Publications of the Astronomical Society of the Pacific, 115:22-36, 2003 January

(C) 2003. The Astronomical Society of the Pacific. All rights reserved. Printed in U.S.A.

\title{
A Search for Cool Subdwarfs: Stellar Parameters for 134 Candidates $^{1}$
}

\author{
David Yong ANd David L. LAMBerT \\ Department of Astronomy, University of Texas, Austin, TX 78712; tofu@ astro.as.utexas.edu, dll@astro.as.utexas.edu \\ Received 2002 September 3; accepted 2002 September 16; published 2002 December 4
}

\begin{abstract}
The results of a search for cool subdwarfs are presented. Kinematic $(U, V$, and $W)$ and stellar parameters $\left(T_{\text {eff }}, \log g,[\mathrm{Fe} / \mathrm{H}]\right.$, and $\left.\xi_{t}\right)$ are derived for 134 candidate subdwarfs based on high-resolution spectra. The observed stars span $4200 \mathrm{~K}<T_{\text {eff }}<6400 \mathrm{~K}$ and $-2.70<[\mathrm{Fe} / \mathrm{H}]<0.25$ including only eight giants $(\log g$ $<4.0$ ). Of the sample, 100 stars have $\mathrm{MgH}$ bands present in their spectra. The targets were selected by their large reduced proper motion, by the offset from the solar-metallicity main sequence, or from the literature. We confirm the claims made by Ryan that the NLTT catalog is a rich source of subdwarfs and verify the success of the reduced proper-motion constraint in identifying metal-poor stars.
\end{abstract}

\section{INTRODUCTION}

The driving force behind our understanding of the chemical evolution of the Galaxy is the interpretation of observed abundance ratios. Self-consistent analyses of large high-quality data sets have revealed detailed abundance patterns (e.g., Edvardsson et al. 1993; McWilliam et al. 1995). Recent attempts to understand the observed abundance trends include endeavors by Timmes, Woosley, \& Weaver (1995), Goswami \& Prantzos (2000), and Alibés, Labay, \& Canal (2001), who predict the evolution of the abundances of all elements from carbon through zinc. Cool stars provide a unique opportunity to test directly these models of Galactic chemical evolution through the abundances of low ionization potential trace elements (e.g., $\mathrm{Rb}, \mathrm{Cs})$ and isotopic ratios measured from molecular bands (e.g., $\mathrm{Mg}$ from $\mathrm{MgH}$, Ti from TiO). Presently, these tests cannot be carried out because of the dearth of known cool metal-poor stars. In order to rectify this situation, we have commenced a search for cool subdwarfs, unevolved metal-poor stars that fall below the solar-metallicity main sequence in color-magnitude diagrams.

Searches undertaken by Carney \& Latham (1987) and Ryan (1989) selected candidates drawn from proper-motion catalogs. These searches were successful in identifying metal-poor dwarfs for subsequent detailed abundance analysis. However, both searches neglected cool subdwarfs. An alternative to searches for metal-poor stars based on proper-motion catalogs is the objective prism surveys, which identify candidate metalpoor stars by the weakening of $\mathrm{Ca}$ II $\mathrm{H}$ and K. Such studies have been conducted by Bond (1970, 1980), Beers, Preston, \& Shectman $(1985,1992)$, and others. Selection criteria that

\footnotetext{
${ }^{1}$ Based in part on observations obtained with the Hobby-Eberly Telescope, which is a joint project of the University of Texas at Austin, the Pennsylvania State University, Stanford University, Ludwig-Maximillians-Universität München, and Georg-August-Universität Göttingen.
}

rely exclusively upon $\mathrm{Ca}$ features as metallicity indicators strongly bias the sample toward warmer stars. Since metal lines weaken with increasing $T_{\text {eff }}$, a temperature estimate is required before deciding whether lines are abnormally weak. In the absence of temperature indicators, a cool metal-poor star will have $\mathrm{Ca}$ II features comparable to a warmer solar-metallicity star. More current work by Christlieb (2000) on the stellar content of the Hamburg/ESO Survey has dramatically increased the yields of metal-poor stars. Candidate metal-poor stars are identified from their location in $(B-V)-\mathrm{Ca}$ line strength space. By comparing the $\mathrm{Ca}$ line strength between stars of similar $T_{\text {eff }}$, this increases the likelihood that a metal deficiency is the cause of the relative weakening of the $\mathrm{Ca}$ line in a candidate. With the remarkable success rate of $60 \%$ for stars below $[\mathrm{Fe} / \mathrm{H}]=-2.0$, twice as good as the HK survey by Beers and collaborators, we look forward to the results of the Hamburg/ESO Survey when applied to cool subdwarfs.

In this paper, we present the results of our search for cool subdwarfs, stellar $\left(T_{\text {eff }}, \log g,[\mathrm{Fe} / \mathrm{H}]\right.$, and $\left.\xi_{t}\right)$ and kinematic parameters $(U, V$, and $W)$ for 134 stars. Our sample includes 80 stars with no prior metallicity estimates. In $\S 2$, we outline and justify the selection criteria. The observations will be described in $\S 3$ and the analysis in $\S 4$. A discussion will be presented in $\S 5$ and concluding remarks given in $\S 6$.

\section{SELECTION CRITERIA}

Our goal was to find previously unidentified subdwarfs with $4000 \mathrm{~K}<T_{\text {eff }}<4700 \mathrm{~K}$ and $[\mathrm{Fe} / \mathrm{H}]<-1.5$. We modeled our search upon the highly successful effort by Ryan (1989) using the New Luyten Two-Tenths (NLTT) catalog of stars with annual proper motions in excess of $0{ }^{\prime \prime} 18 \mathrm{yr}^{-1}$ (Luyten 1979, 1980; Luyten \& Hughes 1980). Subdwarfs are on plunging orbits, often highly elliptical, resulting in large transverse velocities relative to the local standard of rest (LSR). Subdwarfs are 
therefore overrepresented in proper-motion catalogs. Following Ryan (1989), our primary criterion for ensuring that metal-poor candidates were selected was the reduced proper motion, $H=m_{V}+5 \log \mu+5$, where $m_{V}$ is the apparent magnitude and $\mu$ is the proper motion in $\operatorname{arcsec} \mathrm{yr}^{-1}$. The reduced proper motion can also be expressed as $H=M_{V}+5 \log \nu_{T}-3.37$, where $M_{V}$ is the absolute magnitude and $\nu_{T}$ is the transverse velocity in $\mathrm{km} \mathrm{s}^{-1}$. At a given spectral type, a reduced propermotion constraint rejects stars whose transverse velocities fall below a chosen value. Subdwarfs are less luminous than disk dwarfs at a given color and have larger transverse velocities. Both effects conspire to increase the reduced proper motion of the subdwarf population relative to the disk dwarf population. We adopted Ryan's reduced proper-motion constraint requiring that stars have $H_{R} \geq 10.7+2\left(m_{\mathrm{pg}}-m_{R}\right)$, where $H_{R}=m_{R}+$ $5 \log \mu+5$ using Luyten magnitudes $m_{R}$ and $m_{\mathrm{pg}}$. We imposed a magnitude limit $m_{R} \leq 13.0$ and a declination limit $-40^{\circ} \leq$ $\delta \leq 90^{\circ}$, as our observations were made at McDonald Observatory, where we required a reasonable signal-to-noise ratio (S/N) $\left(60\right.$ pixel $^{-1}$ at $\left.6500 \AA\right)$ in $20-30$ minute exposures. To ensure that we observed cool stars, Luyten color classes $g-k$, $k$, and $k-m$ were selected. Carney \& Latham (1987) and Ryan (1989) previously observed the $g-k$ color class. We included these stars so that we could compare our derived stellar parameters with the Carney and Ryan values. Ryan's photometry suggested that the color classes were not accurate. Stars assigned color class $g-k$ by Luyten actually span late $\mathrm{F}$ to late $\mathrm{K}$. Further, the effect of the ultraviolet excess, the hallmark of a subdwarf, is to camouflage a cool subdwarf as a warmer disk dwarf in the Luyten color system. Altogether, our selection criteria resulted in 4445 NLTT candidate subdwarfs.

Our list was augmented by subdwarfs previously identified in the literature. Our final sources of candidate subdwarfs were color-magnitude diagrams constructed using the Hipparcos (ESA 1997) and Yale (van Altena, Lee, \& Hoffleit 1995) parallaxes. Targets located beneath the solar-metallicity main sequence in the range $0.8<B-V<1.4$ were selected. Subdwarfs have a higher $T_{\text {eff }}$ than disk dwarfs at a given mass, which shifts subdwarfs blueward of the disk main sequence. Reid et al. (2001) demonstrated that in the range $B-V<0.8$, the majority of stars below the disk main sequence in a color-magnitude diagram based on Hipparcos data are not subdwarfs, $[\mathrm{Fe} / \mathrm{H}]>-1.0$. Errors in the colors cause these disk dwarfs to appear as subdwarfs. Our observations and analysis will test whether the Reid et al. (2001) findings are valid when we extend to cooler dwarfs, $0.8<B-V<1.4$. In total, we observed around 230 candidate subdwarfs, although in this paper we are reporting on 134 candidates.

\section{OBSERVATIONS AND DATA REDUCTION}

Table 1 contains the list of candidates observed at McDonald Observatory on the $2.7 \mathrm{~m}$ Harlan J. Smith telescope and on the $9.2 \mathrm{~m}$ Hobby-Eberly telescope (HET) between 1999
November and 2002 April. The $2.7 \mathrm{~m}$ data were obtained using the cross-dispersed echelle spectrometer (Tull et al. 1995) at the coudé $\mathrm{f} / 32.5$ focus with a resolving power of either 30,000 or 60,000 . The detector was a Tektronix CCD with $24 \mu \mathrm{m}^{2}$ pixels in a $2048 \times 2048$ format. For this setting, the spectral coverage was from 3800 to $8900 \AA$ with gaps between the orders beyond $5800 \AA$. The HET data were taken with the Upgraded Fiber Optic Echelle spectrograph (Harlow et al. 1996 ) at a resolving power of 11,000 on a $1024 \times 1024$ CCD. The spectral coverage was from 4500 to $9000 \AA$ with gaps between the orders beyond $7300 \AA$. For observations on both telescopes, wavelength coverage incorporated the $\mathrm{MgH} A-X$ bands near $5140 \AA$ as well as the $\mathrm{TiO}(0,0)$ bandhead of the $\gamma$-system $A^{3} \Phi-X^{3} \Delta$ near $7054 \AA$. Visual inspection of the spectra readily indicated the presence or absence of the $\mathrm{MgH}$ or TiO molecular features (see Figs. 1 and 2). Of our sample of 134 stars, 34 displayed neither $\mathrm{MgH}$ nor TiO bands and 100 showed $\mathrm{MgH}$ or $\mathrm{MgH}$ and $\mathrm{TiO}$ bands. Numerous Fe I, Fe II, $\mathrm{Ti} \mathrm{I}$, and $\mathrm{Ti}$ II lines were available in the observed spectra for spectroscopic determination of stellar parameters. For each star, exposure times were generally 20-30 minutes and only occasionally were multiple exposures taken and co-added. Although varying from star to star, the typical $\mathrm{S} / \mathrm{N}$ of the extracted one-dimensional spectra was 60 pixel $^{-1}$ at $6500 \AA$. Onedimensional wavelength-calibrated normalized spectra were extracted in the standard way using the $\mathrm{IRAF}^{2}$ package of programs. Equivalent widths (EWs) were measured using IRAF, where in general Gaussian profiles were fitted to the observed profile. Figure 3 shows the spectra of two different stars, one taken with $R=60,000$ and the other at $R=11,000$, where two Fe I lines used in the analysis are indicated. Although less than ideal, an equivalent width analysis of $R=11,000$ spectra is feasible.

\section{ANALYSIS}

\subsection{Deriving Stellar Parameters}

The LTE stellar line analysis program MOOG (Sneden 1973) was used in combination with the adopted model atmosphere. For $\log g>3.5$, we used the NEXTGEN model atmosphere grid for low-mass stars computed by Hauschildt, Allard, \& Baron (1999). For giants with $\log g \leq 3.5$, we used LTE model atmospheres computed by Kurucz (1993). In both cases we interpolated within the grid when necessary. We derived the model parameters in the following way. We measured the equivalent widths of $\sim 35 \mathrm{Fe}$ I and around five $\mathrm{Fe}$ II lines. Values for $T_{\text {eff }}$ were set from excitation equilibrium; $T_{\text {eff }}$ was adjusted so that $\mathrm{Fe}$ abundances derived from individual lines were independent of excitation potential, as they must be. The Fe abundances derived from individual $\mathrm{Fe}$ I lines must be inde-

\footnotetext{
${ }^{2}$ IRAF is distributed by the National Optical Astronomy Observatory, which is operated by the Association of Universities for Research in Astronomy, Inc., under cooperative agreement with the National Science Foundation.
} 
TABLE 1

Basic Data and Derived Parameters for Objects

\begin{tabular}{|c|c|c|c|c|c|c|c|c|c|c|c|c|c|c|c|}
\hline Name & $\begin{array}{c}\text { R.A. } \\
\text { (J2000.0) }\end{array}$ & $\begin{array}{c}\text { Decl. } \\
\text { (J2000.0) }\end{array}$ & Resolving Power ${ }^{\mathrm{a}}$ & $\begin{array}{l}T_{\text {eff }} \\
(\mathrm{K})\end{array}$ & {$[\mathrm{Fe} / \mathrm{H}]$} & $\begin{array}{c}\log g \\
\left(\mathrm{~cm} \mathrm{~s}^{-2}\right)\end{array}$ & $\begin{array}{c}\xi_{t} \\
\left(\mathrm{~km} \mathrm{~s}^{-1}\right)\end{array}$ & $\begin{array}{c}U_{\mathrm{LSR}} \\
\left(\mathrm{km} \mathrm{s}^{-1}\right)\end{array}$ & $\begin{array}{c}\sigma_{U} \\
\left(\mathrm{~km} \mathrm{~s}^{-1}\right)\end{array}$ & $\begin{array}{c}V_{\mathrm{LSR}} \\
\left(\mathrm{km} \mathrm{s}^{-1}\right)\end{array}$ & $\begin{array}{c}\sigma_{V} \\
\left(\mathrm{~km} \mathrm{~s}^{-1}\right)\end{array}$ & $\begin{array}{c}W_{\mathrm{LSR}} \\
\left(\mathrm{km} \mathrm{s}^{-1}\right)\end{array}$ & $\begin{array}{c}\sigma_{W} \\
\left(\mathrm{~km} \mathrm{~s}^{-1}\right)\end{array}$ & $\begin{array}{l}\text { Luyten } \\
\text { Color or } \\
\text { Source }\end{array}$ & $\begin{array}{l}\text { Molecular } \\
\text { Features? }\end{array}$ \\
\hline PLX $5805 \ldots \ldots$ & 000155 & 260015 & 30,000 & 4600 & -1.72 & 4.25 & 1.0 & 120 & 10 & -97 & 3 & -26 & 8 & Yale & $\mathrm{Y}$ \\
\hline LP 524-64 & 000800 & $\begin{array}{lll}08 & 16 & 42\end{array}$ & 30,000 & 4600 & -1.04 & 5.00 & 1.0 & -90 & 30 & -95 & 25 & 8 & 13 & $k-m$ & $\mathrm{Y}$ \\
\hline LP $646-24$. & 005006 & -042600 & 30,000 & 4800 & -1.01 & 4.50 & 0.7 & -16 & 5 & -120 & 45 & -38 & 16 & $g-k$ & $\mathrm{Y}$ \\
\hline G72-34. & 014604 & 355448 & 11,000 & 4800 & -2.23 & 4.75 & 0.9 & 45 & 10 & -265 & 165 & -243 & 230 & $g-k$ & $\mathrm{~N}$ \\
\hline $\mathrm{BD}-4^{\circ} 290$ & 015218 & -032643 & 60,000 & 4900 & -0.01 & 4.50 & 0.7 & 50 & 10 & -2 & 1 & -10 & 5 & $k$ & $\mathrm{Y}$ \\
\hline $\mathrm{BD}+8^{\circ} 335$ & 021120 & 093724 & 60,000 & 5100 & -0.98 & 4.50 & 0.7 & -35 & 2 & -65 & 13 & -54 & 5 & $g-k$ & $\mathrm{Y}$ \\
\hline HIP 10412 & 021411 & $\begin{array}{lll}01 & 33 & 12\end{array}$ & 30,000 & 4600 & -0.40 & 4.75 & 1.2 & -65 & 6 & -24 & 5 & 2 & 3 & Yale & $\mathrm{Y}$ \\
\hline $\mathrm{BD}+4^{\circ} 415$ & 023435 & 052647 & 60,000 & 4750 & -0.63 & 4.50 & 0.6 & 122 & 6 & -52 & 3 & -15 & 6 & $k$ & $\mathrm{Y}$ \\
\hline G78-26 .... & 031627 & 380556 & 30,000 & 4200 & -1.01 & 4.75 & 0.6 & 65 & 5 & -245 & 15 & -17 & 6 & Gizis 97 & $\mathrm{Y}$ \\
\hline GJ $1064 \mathrm{~A}$ & 034702 & 412538 & 60,000 & 5000 & -1.15 & 4.50 & 0.6 & -106 & 3 & -109 & 7 & -71 & 4 & FJ98 & $\mathrm{Y}$ \\
\hline GJ 1064B & 034703 & 412542 & 60,000 & 4800 & -1.15 & 4.50 & 0.6 & -105 & 6 & -130 & 17 & -80 & 9 & FJ98 & $\mathrm{Y}$ \\
\hline G175-7. & 034828 & 511406 & 11,000 & 5000 & -1.76 & 4.00 & 1.0 & -164 & 240 & -515 & 400 & -23 & 30 & $g-k$ & $\mathrm{~N}$ \\
\hline BD $-4^{\circ} 680$ & 035154 & -034911 & 11,000 & 5800 & -1.89 & 4.00 & 1.0 & -232 & 40 & -185 & 58 & 77 & 58 & $g-k$ & $\mathrm{~N}$ \\
\hline GL $158 \ldots \ldots \ldots$ & 040315 & 351624 & 60,000 & 4800 & -1.79 & 5.00 & 1.0 & -50 & 20 & -185 & 4 & 26 & 1 & FJ98 & $\mathrm{Y}$ \\
\hline G39-36. & 044807 & 330936 & 60,000 & 4200 & -2.50 & 4.00 & 1.0 & -14 & 8 & -95 & 25 & 82 & 20 & $g-k$ & $\mathrm{Y}$ \\
\hline G81-41. & 045505 & 454405 & 60,000 & 4550 & -0.96 & 4.50 & 0.6 & -8 & 4 & -79 & 12 & 72 & 11 & $k$ & $\mathrm{Y}$ \\
\hline $\mathrm{BD}-10^{\circ} 1085$ & 050422 & -100859 & 60,000 & 5100 & -0.50 & 4.50 & 0.8 & -6 & 3 & -70 & 4 & -27 & 1 & $g-k$ & $\mathrm{Y}$ \\
\hline $\mathrm{BD}+19^{\circ} 869$. & 051253 & 194320 & 60,000 & 4600 & -1.01 & 4.50 & 0.5 & 0 & 1 & -110 & 9 & -5 & 1 & $k$ & $\mathrm{Y}$ \\
\hline PLX $1219 \ldots$ & 052310 & 331130 & 30,000 & 4600 & -1.79 & 4.00 & 0.6 & -240 & 15 & -125 & 90 & 9 & 5 & Yale & $\mathrm{Y}$ \\
\hline G101-34 . & 062004 & 382044 & 60,000 & 5000 & -1.50 & 2.75 & 1.0 & -336 & 374 & -1425 & 4930 & 109 & 206 & $g^{\mathrm{b}}$ & $\mathrm{N}$ \\
\hline HIP 30567 & 062530 & 484340 & 60,000 & 4700 & -0.60 & 4.75 & 0.6 & 8 & 4 & -104 & 12 & -46 & 5 & $\begin{array}{c}b \\
k\end{array}$ & $\mathrm{Y}$ \\
\hline G103-50 ... & 064008 & 282712 & 60,000 & 4700 & -2.20 & 4.50 & 0.4 & -147 & 38 & -71 & 3 & 15 & 24 & AAM96, CLLA94 & $\mathrm{Y}$ \\
\hline $\mathrm{AC}+78^{\circ} 2199$ & 065249 & 781230 & 60,000 & 5000 & -0.03 & 4.50 & 0.8 & -120 & 19 & -4 & 4 & -114 & 23 & $g-k$ & $\mathrm{Y}$ \\
\hline G88-1 ......... & 065828 & 185949 & 60,000 & 4600 & -0.84 & 5.00 & 1.0 & 2 & 3 & -45 & 6 & 41 & 4 & $g-k$ & $\mathrm{Y}$ \\
\hline $\mathrm{BD}-17^{\circ} 1716 \ldots$ & 065929 & $-17 \quad 1517$ & 60,000 & 4600 & -0.08 & 4.50 & 0.4 & -53 & 3 & 44 & 3 & -25 & 3 & ${ }_{k}^{k}$ & $\mathrm{Y}$ \\
\hline G87-27 ....... & 071008 & 371634 & 60,000 & 5100 & -0.61 & 3.50 & 0.9 & 20 & 8 & -230 & 76 & -90 & 25 & CLLA94 & $\mathrm{Y}$ \\
\hline G87-35. & 072044 & 292048 & 30,000 & 5300 & -0.53 & 4.50 & 0.8 & -51 & 5 & -123 & 21 & 50 & 6 & $g-k$ & $\mathrm{Y}$ \\
\hline G193-42 & 072222 & 503318 & 11,000 & 5000 & -0.94 & 4.25 & 1.0 & -155 & 35 & -275 & 140 & 37 & 10 & $g-k$ & $\mathrm{~N}$ \\
\hline G193-49. & 072722 & 522612 & 30,000 & 5000 & -0.39 & 5.00 & 0.9 & -72 & 26 & 35 & 14 & -118 & 58 & $k$ & $\mathrm{Y}$ \\
\hline G90-4. & 073023 & 271618 & 11,000 & 5600 & -0.81 & 4.50 & 1.0 & 7 & 10 & -150 & 120 & -108 & 80 & $g-k$ & $\mathrm{~N}$ \\
\hline GSC $03790-02030 \ldots \ldots$ & 074534 & 565722 & 30,000 & 5600 & -1.16 & 4.00 & 0.7 & -43 & 37 & -84 & 50 & -59 & 32 & $\mathrm{~K}$ & $\mathrm{~N}$ \\
\hline LP $208-60 \ldots \ldots \ldots$ & 082144 & 384713 & 11,000 & 5800 & -0.22 & 4.00 & 1.0 & 50 & 35 & -280 & 255 & 34 & 37 & $g-k$ & $\mathrm{~N}$ \\
\hline G115-1. & $\begin{array}{lll}08 & 22 & 17\end{array}$ & 410424 & 30,000 & 4800 & -1.33 & 4.50 & 0.6 & -75 & 3 & -84 & 23 & 34 & 4 & $g-k$ & $\mathrm{Y}$ \\
\hline LP $545-17$ & $08 \quad 2540$ & $\begin{array}{llll}05 & 15 & 28\end{array}$ & 11,000 & 5200 & -1.01 & 4.75 & 1.0 & 52 & 105 & -95 & 50 & 177 & 120 & $k-m$ & $\mathrm{~N}$ \\
\hline PLX 2019 & 082941 & 014448 & 30,000 & 4600 & -1.72 & 4.50 & 1.0 & 237 & 101 & -334 & 104 & -11 & 14 & Yale & Y \\
\hline PLX 2023.01 & 083000 & -095401 & 30,000 & 4400 & -0.39 & 3.90 & 1.0 & -12 & 1 & 4 & 1 & 10 & 1 & Yale & $\mathrm{Y}$ \\
\hline LP 209-14. & $\begin{array}{llll}08 & 31 & 10\end{array}$ & 421348 & 11,000 & 5400 & -1.36 & 4.75 & 1.0 & -155 & 16 & -270 & 265 & 92 & 15 & $\mathrm{~K}$ & $\mathrm{~N}$ \\
\hline G113-49 $\ldots$ & 083512 & 032800 & 11,000 & 5100 & -0.83 & 4.00 & 1.0 & 95 & 120 & -180 & 110 & 77 & 35 & $k-m$ & $\mathrm{~N}$ \\
\hline G9-31 ... & 085245 & 223330 & 11,000 & 5600 & -1.00 & 4.25 & 1.0 & -96 & 16 & -150 & 45 & -93 & 45 & $g-k$ & $\mathrm{~N}$ \\
\hline LP $36-78$ & 085629 & $7058 \quad 14$ & 60,000 & 5150 & 0.25 & 4.50 & 0.7 & -87 & 16 & -56 & 23 & 31 & 2 & $k$ & $\mathrm{Y}$ \\
\hline LP 786-61 .. & 090514 & -183206 & 60,000 & 5800 & -0.76 & 4.00 & 0.8 & 63 & 23 & 16 & 3 & 47 & 20 & $g-k$ & $\mathrm{~N}$ \\
\hline Ross 94 .. & 094722 & 261813 & 60,000 & 4600 & 0.00 & 3.50 & 1.0 & -24 & 2 & -49 & 8 & -18 & 4 & $k$ & Y \\
\hline
\end{tabular}


TABLE 1 (Continued)

\begin{tabular}{|c|c|c|c|c|c|c|c|c|c|c|c|c|c|c|c|}
\hline Name & $\begin{array}{c}\text { R.A. } \\
\text { (J2000.0) }\end{array}$ & $\begin{array}{c}\text { Decl. } \\
\text { (J2000.0) }\end{array}$ & Resolving Power ${ }^{\mathrm{a}}$ & $\begin{array}{l}T_{\text {eff }} \\
(\mathrm{K})\end{array}$ & {$[\mathrm{Fe} / \mathrm{H}]$} & $\begin{array}{c}\log g \\
\left(\mathrm{~cm} \mathrm{~s}^{-2}\right)\end{array}$ & $\begin{array}{c}\xi_{t} \\
\left(\mathrm{~km} \mathrm{~s}^{-1}\right)\end{array}$ & $\begin{array}{c}U_{\mathrm{LSR}} \\
\left(\mathrm{km} \mathrm{s}^{-1}\right)\end{array}$ & $\begin{array}{c}\sigma_{U} \\
\left(\mathrm{~km} \mathrm{~s}^{-1}\right)\end{array}$ & $\begin{array}{c}V_{\mathrm{LSR}} \\
\left(\mathrm{km} \mathrm{s}^{-1}\right)\end{array}$ & $\begin{array}{c}\sigma_{V} \\
\left(\mathrm{~km} \mathrm{~s}^{-1}\right)\end{array}$ & $\begin{array}{c}W_{\mathrm{LSR}} \\
\left(\mathrm{km} \mathrm{s}^{-1}\right)\end{array}$ & $\begin{array}{c}\sigma_{W} \\
\left(\mathrm{~km} \mathrm{~s}^{-1}\right)\end{array}$ & $\begin{array}{c}\text { Luyten } \\
\text { Color or } \\
\text { Source }\end{array}$ & $\begin{array}{c}\text { Molecular } \\
\text { Features? }\end{array}$ \\
\hline G116-55 & 094819 & 340712 & 30,000 & 5600 & -2.10 & 4.00 & 1.0 & -190 & 90 & -165 & 130 & -38 & 90 & $g-k$ & $\mathrm{~N}$ \\
\hline G43-7... & 095013 & 050905 & 30,000 & 4950 & -1.02 & 4.50 & 0.8 & 113 & 19 & -41 & 11 & 12 & 5 & $g-k$ & Y \\
\hline G161-84 & 095139 & -035000 & 60,000 & 4700 & -1.31 & 4.75 & 0.4 & -199 & 53 & -96 & 17 & 131 & 15 & CLLA94 & $\mathrm{Y}$ \\
\hline LP 788-55 & 095433 & -192100 & 60,000 & 4700 & -0.53 & 4.50 & 0.7 & 66 & 18 & -4 & 6 & -5 & 4 & $g-k$ & $\mathrm{Y}$ \\
\hline Wolf 334 & 095744 & 323654 & 11,000 & 5200 & -1.71 & 4.50 & 1.0 & -450 & 320 & -375 & 335 & -163 & 300 & $g-k$ & $\mathrm{~N}$ \\
\hline LP $315-12$. & 095746 & 264522 & 60,000 & 5100 & -0.98 & 4.50 & 0.6 & -122 & 15 & -12 & 4 & -14 & 11 & $g-k$ & Y \\
\hline LP 429-17. & 100108 & 141842 & 60,000 & 4750 & -0.10 & 4.50 & 0.4 & 47 & 10 & -47 & 13 & -15 & 3 & $g-\hat{k}$ & $\mathrm{Y}$ \\
\hline $\mathrm{BD}+53^{\circ} 1395$ & 101357 & 523024 & 60,000 & 4500 & -1.04 & 4.50 & 0.5 & 16 & 1 & -79 & 3 & 12 & 1 & $k$ & $\mathrm{Y}$ \\
\hline $\mathrm{BD}+12^{\circ} 2201$ & 102220 & 120845 & 60,000 & 4500 & -0.23 & 4.50 & 0.9 & -57 & 3 & -17 & 1 & -9 & 2 & $k$ & $\mathrm{Y}$ \\
\hline LP $790-19 \ldots$ & 102607 & -175843 & 60,000 & 4300 & -0.51 & 5.00 & 0.6 & -111 & 7 & -46 & 1 & 9 & 2 & $k$ & $\mathrm{Y}$ \\
\hline $\mathrm{BD}-9^{\circ} 3104$ & 103529 & -102236 & 30,000 & 4900 & -0.60 & 4.50 & 0.8 & 130 & 32 & -82 & 14 & -9 & 9 & $k$ & $\mathrm{Y}$ \\
\hline G119-21 & 103725 & 285548 & 30,000 & 4600 & -0.48 & 4.50 & 0.6 & -81 & 24 & 58 & 15 & -42 & 10 & $k$ & $\mathrm{Y}$ \\
\hline $\mathrm{BD}+31^{\circ} 2175$ & 104016 & 304855 & 60,000 & 4800 & -0.17 & 4.75 & 1.1 & -90 & 4 & -35 & 2 & -13 & 3 & $k$ & $\mathrm{Y}$ \\
\hline PLX 2529.1.. & 105203 & -000938 & 60,000 & 4800 & -0.58 & 4.50 & 0.5 & 39 & 3 & -6 & 3 & $\begin{array}{l}1 \\
-52\end{array}$ & 2 & Yale, HIP & $\mathrm{Y}$ \\
\hline G119-45 ... & 105721 & 305948 & 30,000 & 4800 & 0.14 & 4.50 & 0.7 & -177 & 39 & -85 & 22 & -53 & 20 & $k$ & $\mathrm{Y}$ \\
\hline G58-36 . & 110313 & 220024 & 30,000 & 4600 & -0.05 & 4.50 & 0.8 & -115 & 35 & -47 & 15 & 15 & 16 & $k$ & $\mathrm{Y}$ \\
\hline G253-46 & 110806 & 822454 & 60,000 & 5200 & -0.57 & 4.50 & 0.7 & 0 & 2 & -35 & 6 & 56 & 7 & $\stackrel{\kappa}{g-k}$ & $\mathrm{Y}$ \\
\hline $\mathrm{BD}-10^{\circ} 3216$ & 111111 & -105703 & 60,000 & 4500 & -1.19 & 4.50 & 0.4 & -122 & 3 & -16 & 1 & 34 & 1 & $\hat{k}-\hat{m}$ & $\mathrm{Y}$ \\
\hline HIP $55128 \ldots$ & 111712 & $\begin{array}{ll}1729 & 27\end{array}$ & 30,000 & 4900 & -0.38 & 4.50 & 0.9 & -124 & 26 & -55 & 14 & -53 & 12 & $k$ & $\mathrm{Y}$ \\
\hline LP 792-12. & 111858 & -171548 & 60,000 & 4800 & -0.39 & 5.00 & 0.6 & -77 & 14 & -34 & 2 & 35 & 2 & $\stackrel{n}{g-k}$ & $\mathrm{Y}$ \\
\hline $\mathrm{BD}+15^{\circ} 2325$ & 112219 & 142644 & 60,000 & 4800 & -0.58 & 4.50 & 0.6 & -125 & 12 & -46 & 5 & -9 & 5 & $k$ & $\mathrm{Y}$ \\
\hline G122-22 $\ldots$ & 112400 & 453234 & 30,000 & 4400 & -1.36 & 4.50 & 0.9 & 63 & 15 & -36 & 8 & 58 & 8 & $k$ & $\mathrm{Y}$ \\
\hline G163-80 & 112507 & -055624 & 30,000 & 4700 & 0.05 & 4.75 & 0.6 & -114 & 23 & -55 & 9 & 0 & 8 & $k$ & $\mathrm{Y}$ \\
\hline Ross 109 & $\begin{array}{ll}112715\end{array}$ & 593318 & 60,000 & 5300 & -1.53 & 4.00 & 0.6 & -350 & 300 & -645 & 700 & 217 & 140 & $\mathrm{~K}$ & $\mathrm{~N}$ \\
\hline LP 733-14 .... & $\begin{array}{lll}113829 & \end{array}$ & $\begin{array}{r}-135006 \\
\end{array}$ & 60,000 & 4700 & -0.57 & 4.75 & 0.7 & -15 & 3 & -35 & 11 & -41 & 10 & $g-k$ & Y \\
\hline LP 734-54 ... & 115754 & -094848 & 60,000 & 4800 & -0.66 & 4.50 & 0.4 & -78 & 13 & -15 & 4 & 5 & 2 & $g-\hat{k}$ & $\mathrm{Y}$ \\
\hline Wolf 1424 & 120019 & 203543 & 60,000 & 4600 & -1.19 & 4.50 & 0.4 & -28 & 4 & -120 & 25 & -33 & 6 & $k$ & $\mathrm{Y}$ \\
\hline LP 734-101 & 121655 & $\begin{array}{r}-114906 \\
-\end{array}$ & 60,000 & 5500 & 0.06 & 4.50 & 0.7 & $\begin{array}{l}-20 \\
-86\end{array}$ & 16 & -36 & 10 & -9 & 4 & $\stackrel{\kappa}{g-k}$ & $\mathrm{Y}$ \\
\hline G13-29 .... & 121920 & 022642 & 30,000 & 4800 & -0.66 & 4.50 & 0.6 & -6 & 4 & -101 & 30 & -8 & 15 & $\begin{array}{l}g \\
g-k\end{array}$ & $\mathrm{Y}$ \\
\hline G198-61 & 123523 & 374430 & 30,000 & 5300 & -0.12 & 4.50 & 1.0 & 176 & 36 & -42 & 11 & 21 & 7 & $k$ & $\mathrm{Y}$ \\
\hline G149-9. & 124803 & 274548 & 30,000 & 4900 & -0.62 & 4.75 & 0.8 & 24 & 16 & -315 & 150 & -86 & 1 & $k$ & $\mathrm{Y}$ \\
\hline HIP 62627 & 124956 & 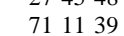 & 60,000 & 4800 & $\begin{array}{l}-0.24 \\
-0.24\end{array}$ & 5.00 & $\begin{array}{l}1.0 \\
1.2\end{array}$ & -8 & 2 & -93 & 3 & -17 & 2 & $\begin{array}{c}\kappa \\
\text { HIP }\end{array}$ & $\mathrm{Y}$ \\
\hline G149-18 & 125246 & 222700 & 30,000 & 5000 & -0.03 & 4.50 & 0.8 & -214 & 44 & -33 & 11 & 29 & 2 & $k$ & $\mathrm{Y}$ \\
\hline W453 & 125749 & 054554 & 30,000 & 4600 & -0.04 & 4.75 & 0.9 & -71 & 17 & -38 & 13 & 2 & 2 & $k$ & $\mathrm{Y}$ \\
\hline HD 114095 & 130826 & -071830 & 60,000 & 4730 & -0.71 & 2.40 & 1.3 & 65 & 43 & -290 & 166 & -49 & 32 & AAM96 & $\mathrm{Y}$ \\
\hline $\mathrm{BD}+68^{\circ} 714$ & 131021 & 672941 & 30,000 & 4900 & -0.05 & 4.50 & 1.0 & -96 & 3 & -70 & 2 & 7 & 1 & $\mathrm{~K} 1$ & $\mathrm{Y}$ \\
\hline Ross 466 . & 131514 & -110112 & 30,000 & 5000 & -0.86 & 4.75 & 0.9 & -71 & 17 & -115 & 65 & -103 & 30 & $g-k$ & $\mathrm{Y}$ \\
\hline LHS 2715 & 131857 & -030418 & 60,000 & 4400 & -1.56 & 4.00 & 0.6 & -40 & 7 & -120 & 7 & 115 & 1 & Gizis97 & $\mathrm{Y}$ \\
\hline LP $172-89$ & 132638 & 464851 & 30,000 & 4800 & -0.49 & 4.50 & 0.6 & -124 & 28 & -34 & 9 & 0 & 2 & $g-k$ & $\mathrm{Y}$ \\
\hline LP 738-45 & 133814 & -154714 & 30,000 & 4400 & -0.12 & 4.50 & 0.8 & -81 & 17 & -28 & 14 & 14 & 4 & ${ }_{k}^{n}$ & $\mathrm{Y}$ \\
\hline G255-44 & 135536 & 740012 & 60,000 & 4900 & -1.30 & 4.50 & 0.7 & -107 & 17 & -25 & 15 & 74 & 7 & $k$ & $\mathrm{Y}$ \\
\hline G65-22. & 140144 & 085517 & 60,000 & 5000 & -1.66 & 4.50 & 0.4 & 10 & 8 & -225 & 76 & -83 & 25 & AAM96, CLLA94 & $\mathrm{Y}$ \\
\hline $\mathrm{BD}+30^{\circ} 2490$ & 141124 & 300502 & 30,000 & 4600 & $\begin{array}{l}1.00 \\
-0.18\end{array}$ & 4.75 & 0.6 & $\begin{array}{l}10 \\
-68\end{array}$ & 5 & -88 & 8 & 35 & 3 & HIVIOC, & $\mathrm{Y}$ \\
\hline G239-12 .... & 141853 & 731412 & 60,000 & 5800 & -2.62 & 4.00 & 1.0 & 74 & 25 & -333 & 160 & 127 & 170 & $\hat{K}$ & $\mathrm{~N}$ \\
\hline HIP 70152 & 142114 & 085816 & 60,000 & 4800 & -0.05 & 5.00 & 1.0 & 50 & 7 & -36 & 5 & -20 & 4 & RWMRKM01 & $\mathrm{Y}$ \\
\hline HIP 70715 & 142745 & 235027 & 60,000 & 4400 & -0.11 & 4.50 & 0.6 & -110 & 9 & -66 & 6 & -9 & 4 & HIP & $\mathrm{Y}$ \\
\hline
\end{tabular}


TABLE 1 (Continued)

\begin{tabular}{|c|c|c|c|c|c|c|c|c|c|c|c|c|c|c|c|}
\hline Name & $\begin{array}{c}\text { R.A. } \\
\text { (J2000.0) }\end{array}$ & $\begin{array}{c}\text { Decl. } \\
\text { (J2000.0) }\end{array}$ & Resolving Power ${ }^{\mathrm{a}}$ & $\begin{array}{l}T_{\text {eff }} \\
(\mathrm{K})\end{array}$ & {$[\mathrm{Fe} / \mathrm{H}]$} & $\begin{array}{c}\log g \\
\left(\mathrm{~cm} \mathrm{~s}^{-2}\right)\end{array}$ & $\begin{array}{c}\xi_{t} \\
\left(\mathrm{~km} \mathrm{~s}^{-1}\right)\end{array}$ & $\begin{array}{c}U_{\mathrm{LSR}} \\
\left(\mathrm{km} \mathrm{s}^{-1}\right)\end{array}$ & $\begin{array}{c}\sigma_{U} \\
\left(\mathrm{~km} \mathrm{~s}^{-1}\right)\end{array}$ & $\begin{array}{c}V_{\mathrm{LSR}} \\
\left(\mathrm{km} \mathrm{s}^{-1}\right)\end{array}$ & $\begin{array}{c}\sigma_{V} \\
\left(\mathrm{~km} \mathrm{~s}^{-1}\right)\end{array}$ & 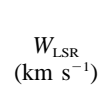 & $\begin{array}{c}\sigma_{W} \\
\left(\mathrm{~km} \mathrm{~s}^{-1}\right)\end{array}$ & $\begin{array}{c}\text { Luyten } \\
\text { Color or } \\
\text { Source }\end{array}$ & $\begin{array}{c}\text { Molecular } \\
\text { Features? }\end{array}$ \\
\hline LP 500-92 & 143413 & 123457 & 30,000 & 4500 & -0.38 & 4.50 & 0.9 & -134 & 9 & -37 & 4 & 10 & 5 & $k-m$ & $\mathrm{Y}$ \\
\hline LP $175-8$. & 143610 & 450859 & 30,000 & 4900 & -0.31 & 4.75 & 0.6 & -123 & 25 & -65 & 14 & 33 & 10 & $k$ & $\mathrm{Y}$ \\
\hline HD 131287 & 145004 & 603936 & 30,000 & 4800 & -0.04 & 4.50 & 1.0 & -4 & 1 & -12 & 1 & -13 & 1 & $k$ & $\mathrm{Y}$ \\
\hline $\mathrm{BD}+23^{\circ} 2751$ & 145342 & 232043 & 60,000 & 4700 & -0.51 & 4.50 & 0.6 & -76 & 2 & -62 & 2 & 20 & 1 & $k$ & $\mathrm{Y}$ \\
\hline LP 502-8 ..... & 150914 & 143123 & 30,000 & 5000 & -0.95 & 4.50 & 0.6 & -80 & 17 & -119 & 25 & 113 & 16 & $g-k$ & Y \\
\hline HIP $74235 \ldots$ & 151013 & -162246 & 60,000 & 4900 & -1.51 & 4.50 & 0.8 & 295 & 2 & -505 & 18 & -62 & 10 & HIP & $\mathrm{Y}$ \\
\hline LP $802-56$. & 151912 & -210046 & 60,000 & 5200 & -0.88 & 4.50 & 0.6 & -143 & 7 & -130 & 32 & -82 & 7 & $k$ & Y \\
\hline $\mathrm{BD}+2^{\circ} 2944$ & 151919 & 014555 & 30,000 & 6000 & -0.02 & 3.50 & 1.8 & 81 & 1 & -8 & 1 & -3 & 1 & K4 & $\mathrm{N}$ \\
\hline Ross $804 \ldots$ & 154529 & -134918 & 30,000 & 4600 & -0.93 & 4.50 & 0.6 & 120 & 85 & -285 & 360 & -108 & 200 & $k$ & Y \\
\hline G225-50 & 161445 & 552549 & 30,000 & 4600 & -0.13 & 4.50 & 1.0 & -60 & 13 & -43 & 6 & 8 & 7 & $g-k$ & Y \\
\hline LP 330-7 & 162306 & 320835 & 30,000 & 4800 & -0.22 & 4.50 & 0.6 & 74 & 16 & 4 & 6 & 10 & 6 & $g-k$ & $\mathrm{Y}$ \\
\hline G202-57 & 163337 & 511330 & 30,000 & 4900 & -1.05 & 4.50 & 0.6 & 180 & 82 & -65 & 32 & 49 & 18 & $g-k$ & $\mathrm{Y}$ \\
\hline G17-25.. & 163442 & -041345 & 60,000 & 4950 & -1.46 & 4.50 & 0.3 & 11 & 11 & -115 & 24 & -29 & 6 & CLLA94 & $\mathrm{Y}$ \\
\hline BD $-14^{\circ} 4454$ & 163614 & -151012 & 30,000 & 4600 & -0.95 & 4.50 & 0.6 & -111 & 2 & -90 & 8 & 35 & 5 & $k$ & $\mathrm{Y}$ \\
\hline LP $445-55 \ldots$. & 164258 & 192209 & 30,000 & 5200 & -1.80 & 4.00 & 0.6 & 118 & 24 & 38 & 8 & 84 & 13 & $g-k$ & $\mathrm{~N}$ \\
\hline LP 447-2. & 170805 & 175738 & 30,000 & 5800 & -1.79 & 4.00 & 0.9 & -146 & 7 & -272 & 26 & 46 & 18 & $g-k$ & $\mathrm{~N}$ \\
\hline LP $747-18$ & 171644 & -130112 & 30,000 & 5800 & -0.95 & 4.50 & 0.8 & 71 & 51 & -322 & 327 & 41 & 29 & $g-k$ & $\mathrm{~N}$ \\
\hline LP $447-34 \ldots$ & 171708 & 180706 & 30,000 & 4800 & -0.35 & 4.50 & 0.9 & 53 & 18 & -45 & 20 & 11 & 4 & $g-k$ & $\mathrm{Y}$ \\
\hline G19-25. & 172559 & -024436 & 60,000 & 4900 & -2.01 & 4.50 & 0.4 & 116 & 86 & -93 & 85 & 9 & 6 & AAM96, CLLA94 & $\mathrm{Y}$ \\
\hline LP $808-11 \ldots \ldots \ldots \ldots$ & 173353 & -165854 & 30,000 & 5500 & -1.23 & 4.00 & 0.7 & -35 & 40 & -225 & 200 & -78 & 65 & $g-k$ & $\mathrm{~N}$ \\
\hline $\mathrm{BD}-8^{\circ} 4501$ & 174728 & -084648 & 30,000 & 5800 & -1.80 & 4.00 & 0.8 & 126 & 12 & -45 & 16 & -156 & 38 & $g-k$ & $\mathrm{~N}$ \\
\hline $\mathrm{G} 204-47 \ldots \ldots$ & 180929 & 471324 & 30,000 & 5200 & -0.88 & 4.50 & 1.0 & 170 & 126 & -137 & 65 & 73 & 59 & $\mathrm{~K}$ & $\mathrm{~N}$ \\
\hline $\mathrm{BD}+5^{\circ} 3640$ & 181222 & 052404 & 60,000 & 4950 & -1.34 & 4.50 & 0.4 & 61 & 13 & -216 & 21 & 38 & 5 & AAM96 & $\mathrm{Y}$ \\
\hline BD $-17^{\circ} 5287$ & 184057 & -170954 & 30,000 & 5800 & -0.70 & 4.50 & 1.0 & -60 & 25 & -155 & 80 & 77 & 35 & $\mathrm{~K}$ & $\mathrm{~N}$ \\
\hline G227-44 $\ldots$ & 184143 & 583430 & 30,000 & 5400 & -1.45 & 4.50 & 1.0 & -314 & 250 & -210 & 11 & -37 & 42 & $\mathrm{~K}$ & $\mathrm{~N}$ \\
\hline G207-23 & 191613 & 370420 & 30,000 & 5000 & -1.90 & 4.00 & 0.6 & -175 & 6 & -319 & 1 & 41 & 15 & $\mathrm{~K}$ & $\mathrm{~N}$ \\
\hline HD 181007 & 191928 & -202540 & 60,000 & 4700 & -1.93 & 1.50 & 1.6 & 65 & 43 & -290 & 166 & -49 & 32 & None $^{c}$ & $\mathrm{~N}$ \\
\hline LP 752-18 ... & 192547 & -110954 & 30,000 & 5800 & -1.00 & 4.00 & 1.0 & 60 & 25 & -75 & 40 & 17 & 10 & $g-k$ & $\mathrm{~N}$ \\
\hline LP 753-29. & 195004 & -131912 & 30,000 & 5400 & -1.88 & 4.00 & 1.0 & -65 & 26 & -155 & 76 & 77 & 35 & $g-k$ & $\mathrm{~N}$ \\
\hline G23-14. & 195150 & 053646 & 60,000 & 5000 & -1.51 & 3.00 & 1.0 & 116 & 86 & -93 & 85 & 9 & 6 & $g^{\mathrm{d}}$ & $\mathrm{N}$ \\
\hline LP 575-39 & 202326 & 055050 & 30,000 & 4700 & -0.42 & 4.50 & 0.6 & -90 & 13 & -11 & 7 & -19 & 12 & $g-k$ & $\mathrm{Y}$ \\
\hline LP 575-40. & 202356 & 052444 & 30,000 & 4800 & -0.10 & 4.50 & 1.0 & -110 & 25 & 20 & 15 & -29 & 18 & $k-m$ & $\mathrm{Y}$ \\
\hline LP $815-43$. & 203814 & -202554 & 30,000 & 6400 & -2.70 & 4.00 & 1.0 & 140 & 140 & -290 & 265 & 36 & 27 & $g-k$ & $\mathrm{~N}$ \\
\hline HD 200968 & 210710 & -135523 & 30,000 & 5000 & -0.16 & 4.50 & 1.2 & -52 & 1 & -14 & 1 & 2 & 1 & KF99 & $\mathrm{Y}$ \\
\hline $\mathrm{BD}+22^{\circ} 4567$ & 221031 & 224749 & 30,000 & 4800 & -0.33 & 4.75 & 1.1 & 75 & 4 & -5 & 1 & 70 & 3 & $k$ & Y \\
\hline $\mathrm{BD}+30^{\circ} 4633$ & 221206 & 313341 & 60,000 & 4600 & -0.01 & 4.50 & 0.8 & 92 & 6 & -35 & 1 & -6 & 2 & $k-m$ & $\mathrm{Y}$ \\
\hline HIP 109801 & 221424 & -084442 & 30,000 & 4600 & -1.70 & 4.50 & 1.0 & 130 & 50 & -225 & 75 & -43 & 25 & HIP & Y \\
\hline Ross 237 & 225330 & 274518 & 30,000 & 4900 & -1.49 & 4.25 & 0.9 & -118 & 25 & -315 & 16 & 8 & 25 & $k$ & Y \\
\hline Ross 242 & 230849 & 270054 & 60,000 & 4700 & -0.94 & 4.50 & 0.4 & 50 & 14 & -130 & 16 & -67 & 30 & $k$ & $\mathrm{Y}$ \\
\hline LP 702-79 & 232332 & -060012 & 30,000 & 4800 & 0.05 & 4.50 & 1.1 & -150 & 60 & -70 & 25 & 1 & 22 & $g-k$ & $\mathrm{Y}$ \\
\hline HIP 115664 & 232556 & 291141 & 30,000 & 4600 & -0.90 & 5.00 & 0.6 & -85 & 16 & -215 & 20 & -78 & 25 & HIP & Y \\
\hline $\mathrm{BD}+28^{\circ} 4634$ & 234510 & 293343 & 60,000 & 5000 & -0.30 & 4.50 & 0.7 & -138 & 3 & 6 & 2 & -60 & 1 & $k$ & Y \\
\hline
\end{tabular}

NOTE. - Units of right ascension are hours, minutes, and seconds, and units of declination are degrees, arcminutes, and arcseconds.

${ }^{2}$ HET data taken with $R=11,000,2.7$ m data taken with $R=30,000$ and 60,000 .

b Star chosen from Carney et al. 1994 and Alonso et al. 1996a, but also in NLTT catalog.

'Star chosen from Alonso et al. 1996a; not in NLTT catalog.

REFERENCES. - AAM96 = Alonso et al. 1996a; CLLA94 = Carney et al. 1994; FJ98 = Fuchs \& Jahreiß 1998; KF99 = Kotoneva \& Flynn 1999, private communication; Gizis97 = Gizis 1997; HIP = Hipparcos color-magnitude diagram; RWMRKM01 = Reid et al. 2001; Yale = Yale color-magnitude diagram. 


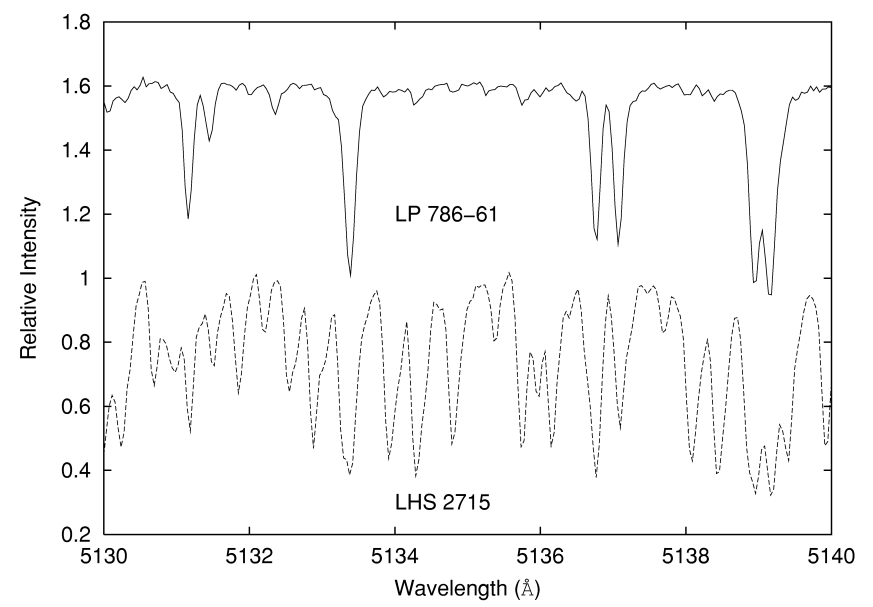

FIG. 1. $-R=60,000$ data showing the absence (top spectrum) and presence (bottom spectrum) of the $\mathrm{MgH} A-X$ lines near $5140 \AA$. Many of the additional lines in the bottom spectrum are due to $\mathrm{MgH}$.

pendent of the strength of the line. Thus, the microturbulence $\xi_{t}$ was estimated. Finally, the gravity was determined from ionization equilibrium. That is, the $\mathrm{Fe}$ abundance derived from $\mathrm{Fe}$ I lines must agree with the abundance derived from Fe II lines. This process was iterated until a consistent set of parameters were obtained (see Fig. 4). Ti I and Ti II lines were also used to check the stellar parameters. The final value of $[\mathrm{Fe} / \mathrm{H}]$ was simply the weighted mean of the Fe I and Fe II abundances

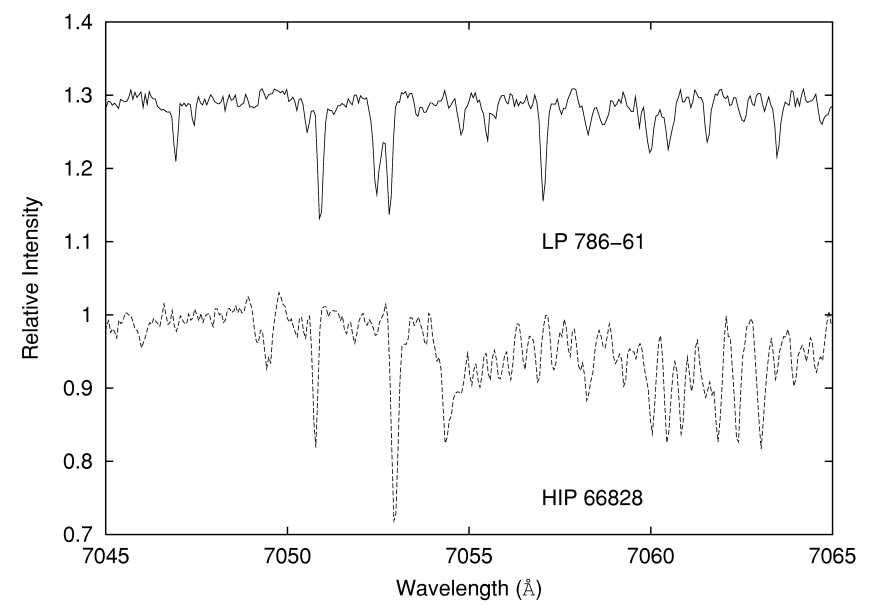

FIG. 2. $-R=60,000$ data showing the absence (top spectrum) and presence (bottom spectrum) of the TiO $\gamma$-system $A^{3} \Phi-X^{3} \Delta$ bandhead near $7054 \AA$.

derived from the accepted model (see Table 1 for model parameters).

The $g f$-values of the $\mathrm{Fe}$ lines used in the analysis were selected from Lambert et al. (1996) and a list compiled by R. E. Luck (1993, private communication). The $g f$-values of the Ti lines were selected from the R. E. Luck compilation. Where possible, only weak lines, EWs $<90 \mathrm{~m} \AA$, were used in the analysis. We checked our analysis techniques by observing

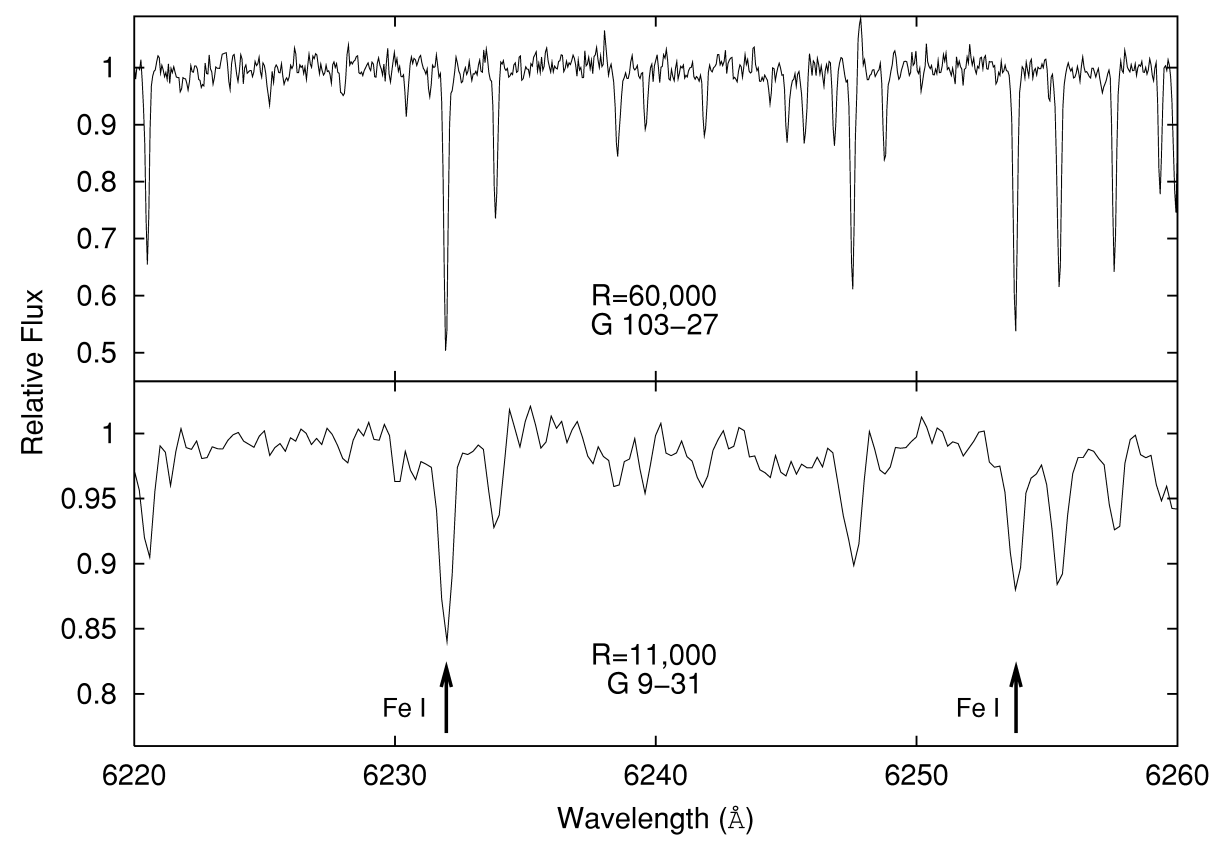

FIG. 3. - Spectra of G103-27 taken at $R=60,000$ and G9-31 taken at $R=11,000$. Both stars have similar stellar parameters. Representative Fe I lines used to derive stellar parameters are highlighted. Importantly, the $R=11,000$ spectrum has Fe I lines for which equivalent widths can be measured. 


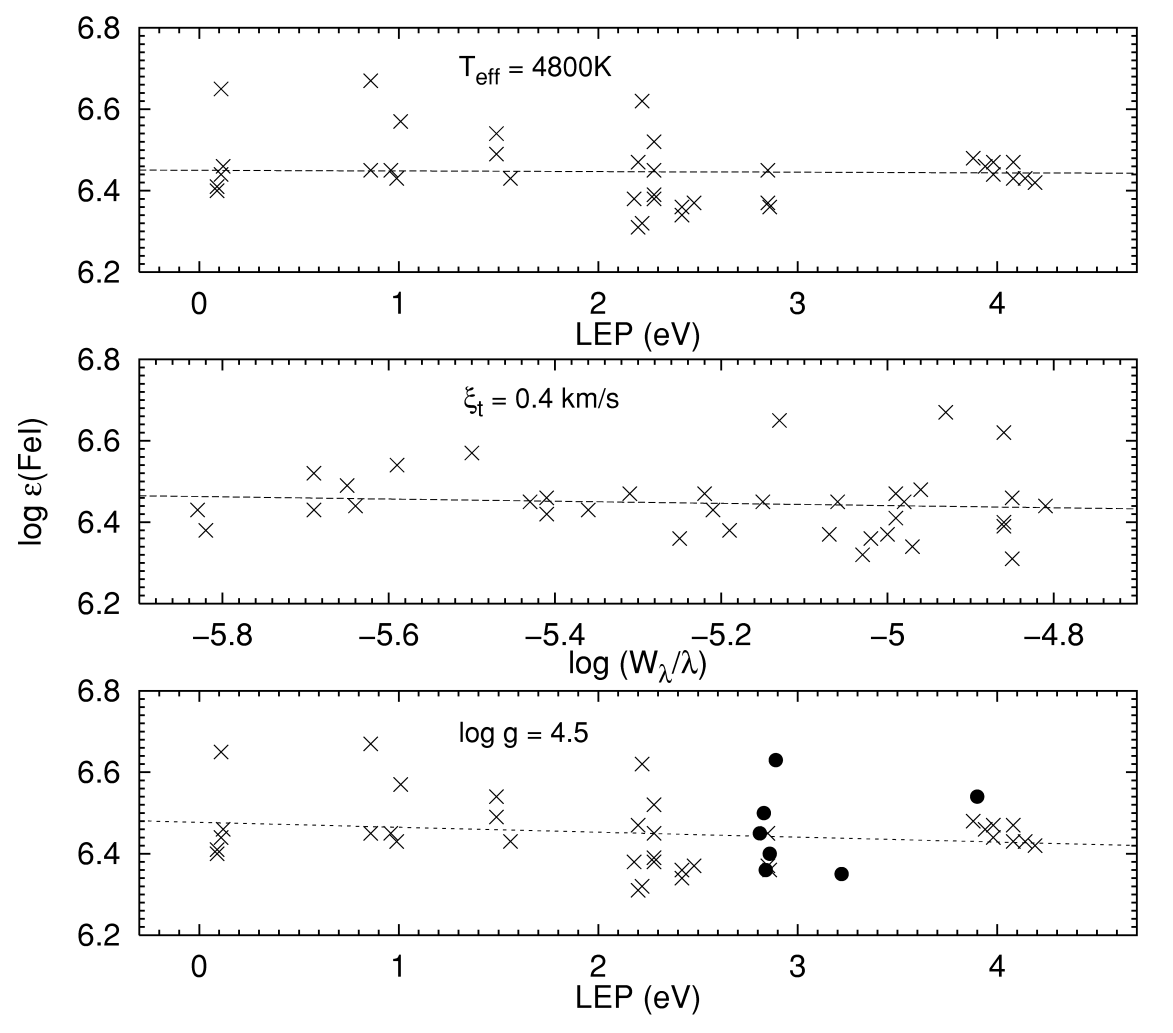

FIG. 4.-Determination of stellar parameters $T_{\text {eff }}, \log g$, and $\xi_{t}$ using excitation and ionization equilibrium for GJ 1064B. In the top panel, the lower excitation potential (LEP)-abundance relation is used to set $T_{\text {eff }}$. In the middle panel, the reduced equivalent width $\left(W_{\lambda} / \lambda\right)$-abundance relation is used to determine $\xi_{t}$. In the bottom panel, the abundances of $\mathrm{Fe}$ I (crosses) and $\mathrm{Fe}$ II (filled circles) are used to fix log $g$. In all panels, the line represents the least-squares fit to the data.

the solar spectrum at $R=60,000$. We measured $30 \mathrm{Fe}$ I lines and seven Fe II lines and compared our equivalent widths with the Grevesse \& Sauval (1999) values. Our equivalent widths were larger by a mean value of $3.7 \mathrm{~m} \AA$ with a standard deviation of $2.6 \mathrm{~m} \AA$. Using our equivalent widths and a NEXTGEN model atmosphere, we derived a solar abundance of $\log \epsilon(\mathrm{Fe})=7.54$. Considering the Grevesse \& Sauval (1999) value of $\log \epsilon(\mathrm{Fe})$ $=7.50 \pm 0.05$ derived from their empirical model solar atmosphere, we adopted $\log \epsilon(\mathrm{Fe})=7.52$ as the solar $\mathrm{Fe}$ abundance for this study.

Our derived model parameters, $T_{\text {eff }}, \log g, \xi_{t}$, and $[\mathrm{Fe} / \mathrm{H}]$, have associated uncertainties. We varied $T_{\text {eff }}$ until the trends between lower excitation potential and abundance were unacceptable. Similarly, we took values for $\xi_{t}$ that noticeably changed the trends between equivalent width and abundance. For $\log g$, we measured the standard deviation of the $\mathrm{Fe}$ abundance as derived from $\mathrm{Fe}$ I lines (typically $0.1-0.15$ dex) and then allowed $\mathrm{Fe} \mathrm{I}$ and $\mathrm{Fe}$ II to agree within this standard deviation. This produced uncertainties of $\delta T_{\text {eff }}=150 \mathrm{~K}$, $\delta \log g=0.3 \mathrm{dex}, \delta \xi_{t}=0.3 \mathrm{~km} \mathrm{~s}^{-1}$, and $\delta[\mathrm{Fe} / \mathrm{H}]=0.2 \mathrm{dex}$ in the model parameters.

\subsection{Comparison with Literature}

For the 134 candidate subdwarfs analyzed here, a search on SIMBAD indicated that there were 37 stars with previously determined values for $T_{\text {eff }}$ and 54 stars with previously determined values for $[\mathrm{Fe} / \mathrm{H}]$. We have compared our values with those found in the literature (see Table 2 and Figs. 5 and 6). We find a mean offset $\left\langle T_{\text {eff }}\right.$ (this study) $-T_{\text {eff }}$ (literature) $\rangle=$ $-25 \mathrm{~K}$ with a standard deviation of $114 \mathrm{~K}$. For $[\mathrm{Fe} / \mathrm{H}]$, the mean offset is $\langle[\mathrm{Fe} / \mathrm{H}]$ (this study) $-[\mathrm{Fe} / \mathrm{H}]($ literature $)\rangle=$ -0.1 dex with a standard deviation of 0.38 dex. The agreement is reasonable between the stellar parameters derived in this study and the values found in a variety of sources in the literature.

Table 2 shows values for $[\mathrm{Fe} / \mathrm{H}]$ given by Ryan \& Norris (1991) for a number of stars. However, these metallicities are not paired with values for $T_{\text {eff }}$. The Ryan \& Norris (1991) sample are reobservations of a subset of metal-weak stars identified in Ryan (1989). An index measuring the strength of the $\mathrm{Ca}$ II $\mathrm{K}$ line was calibrated empirically as a function of $B-V$ to give $[\mathrm{Fe} / \mathrm{H}]$. Alonso, Arribas, \& Martínez-Roger (1996a, 1999a) determined $T_{\text {eff }}$ using the infrared flux method, which 
TABLE 2

COMPARISON WITH LITERATURE

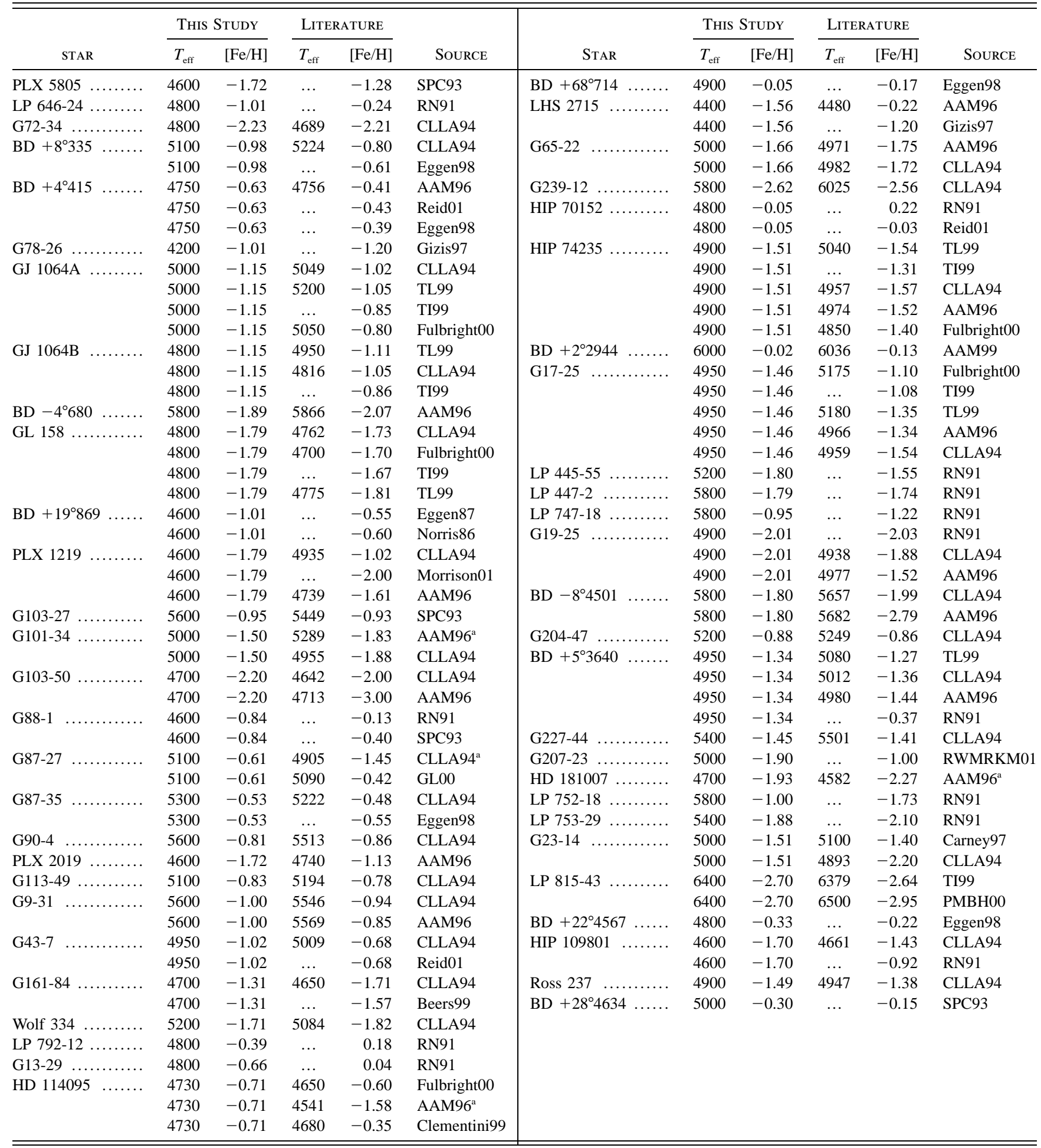

NoTE.-The following references were based on spectroscopic data: Beers99, CLLA94, Carney97, Clementini99, Fulbright00, Gizis97, Primas00, RN91, TI99, and TL99.

${ }^{\text {a }}$ Giant star assumed to be a dwarf.

REFERENCES.-AAM96 = Alonso et al. 1996a; AAM99 = Alonso et al. 1999a; Beers99 = Beers et al. 1999; CLLA94 = Carney et al. 1994; Carney97 $=$ Carney et al. 1997; Clementini99 = Clementini et al. 1999; Eggen87 = Eggen 1987; Eggen98 = Eggen 1998; Fulbright00 = Fulbright $2000 ;$ GL00 = Gay \& Lambert 2000; Gizis97 = Gizis 1997; Morrison01 = Morrison et al. 2001; Norris86 = Norris 1986; Primas00 = Primas et al. 2000; Reid01 $=$ Reid et al. 2001; RN91 = Ryan \& Norris 1991; SPC93 = Schuster, Parrao, \& Contreras Martinez 1993; TI99 = Thévenin \& Idiart 1999; TL99 = Tomkin \& Lambert 1999. 


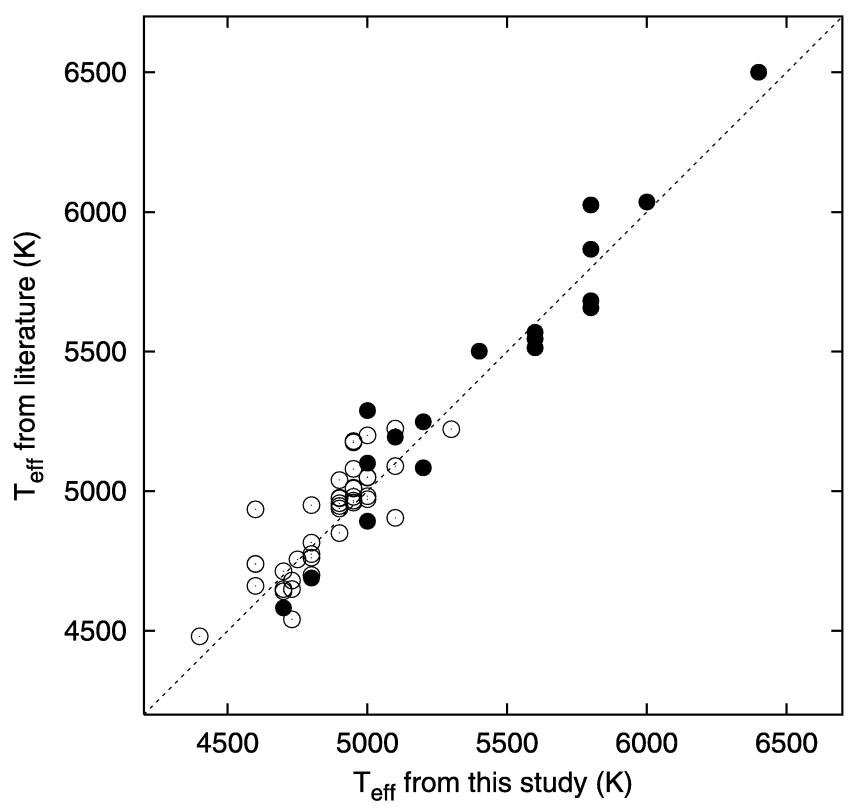

FIG. 5. $-T_{\text {eff }}$ comparisons between this work and various literature studies. The filled circles represent stars that have neither $\mathrm{MgH}$ nor TiO bands in their spectra. The dotted line represents the line of equality.

requires a metallicity and gravity estimate. The derived $T_{\text {eff }}$ are insensitive to the input metallicity and gravity. We also computed $T_{\text {eff }}$ derived by use of the $T_{\text {eff }}:[\mathrm{Fe} / \mathrm{H}]:$ color relations given in Alonso et al. (1996b, 1999b) assuming values for $[\mathrm{Fe} / \mathrm{H}]$ from this study (see Table 3).

$\mathrm{MgH}$ and $\mathrm{TiO}$ bands will not be visible in the spectra of stars with sufficiently high temperatures and/or low abundances. Indeed, this is the case for 34 of the stars as noted in the last column of Table 1 .

\section{DISCUSSION}

\subsection{Kinematics}

We selected stars with large reduced proper motions and therefore expect the observed sample to be kinematically distinct from the thin disk. Following the prescription given by Johnson \& Soderblom (1987), the Galactic space-velocity components $U, V$, and $W$ were calculated along with the associated uncertainties (see Table 1). To correct for the solar motion with respect to the LSR, we assumed the Dehnen \& Binney (1998) values $(-10,+5,+7) \mathrm{km} \mathrm{s}^{-1}$ in $(U, V, W)$. In the absence of Hipparcos parallaxes, spectroscopic parallaxes were determined by using the derived model parameters and the Girardi et al. (2000) isochrones. Figure 7 shows $[\mathrm{Fe} / \mathrm{H}]$ versus $U_{\mathrm{LSR}}$, $V_{\mathrm{LSR}}$, and $W_{\mathrm{LSR}}$. We identify stars that lag the LSR, $V<-50$ $\mathrm{km} \mathrm{s}^{-1}$, as being members of the thick disk or halo. As expected from a reduced proper-motion constraint, we have selected stars that belong to populations kinematically distinct from the thin disk. Many of the observed stars have abundances indicative

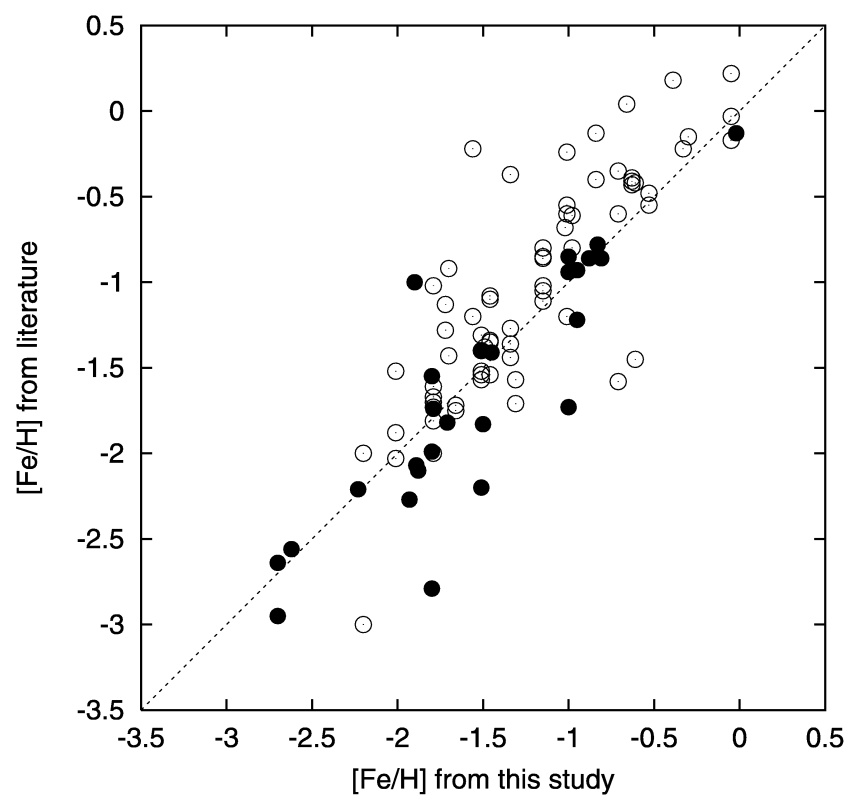

FIG. 6.- $[\mathrm{Fe} / \mathrm{H}]$ comparisons between this work and various literature studies. The filled circles represent stars that have neither $\mathrm{MgH}$ nor $\mathrm{TiO}$ bands in their spectra. The dotted line represents the line of equality. We note that the scatter for the open circles is slightly larger than for the filled circles.

of the thick disk and halo. Ryan (1989) claimed that the NLTT catalog was a rich source of subdwarfs as identified by the ultraviolet excess. Figure 8 shows the numbers of stars for various metallicity bins. Given that 27 stars have $[\mathrm{Fe} / \mathrm{H}] \leq$ -1.5 , our data endorse Ryan's claims. From the kinematics and abundances of the observed stars, we conclude that the reduced proper-motion constraint successfully selected metalweak thick-disk and halo stars.

\subsection{Temperature}

Our goal was to find cool stars, $4000 \mathrm{~K}<T_{\text {eff }}<4700 \mathrm{~K}$. Of the 134 stars presented in this paper, $44 \mathrm{had} T_{\text {eff }} \leq 4700 \mathrm{~K}$ and, given the uncertainties in $T_{\text {eff }}$, we note that 69 of the 134 stars had $T_{\text {eff }} \leq 4800 \mathrm{~K}$. Figure 9 shows the number of stars versus $T_{\text {eff }}$ for this study and the Carney et al. (1994) study. Our sample includes cooler stars than the Carney study, which highlights the different temperature regimes of interest. In addition to the stars presented here, we have observations of a further 100 stars, which are simply too cool for equivalent width analysis. Molecular bands make identification of the continuum virtually impossible, and finding unblended $\mathrm{Fe} \mathrm{I}$ and $\mathrm{Fe}$ II lines is problematic. Given the strength of the TiO bands, we assume that these stars are all cooler than $4500 \mathrm{~K}$. We are exploring various techniques for determination of the stellar parameters. The strength of the $\mathrm{TiO}$ bandheads in some stars suggests rather low values for $T_{\text {eff }}$, thus we reconfirm Ryan's finding regarding Luyten's photometry that "the values tabulated in the NLTT catalog must be regarded as approximate only." 
TABLE 3

COMPARISON OF $T_{\text {eff }}$

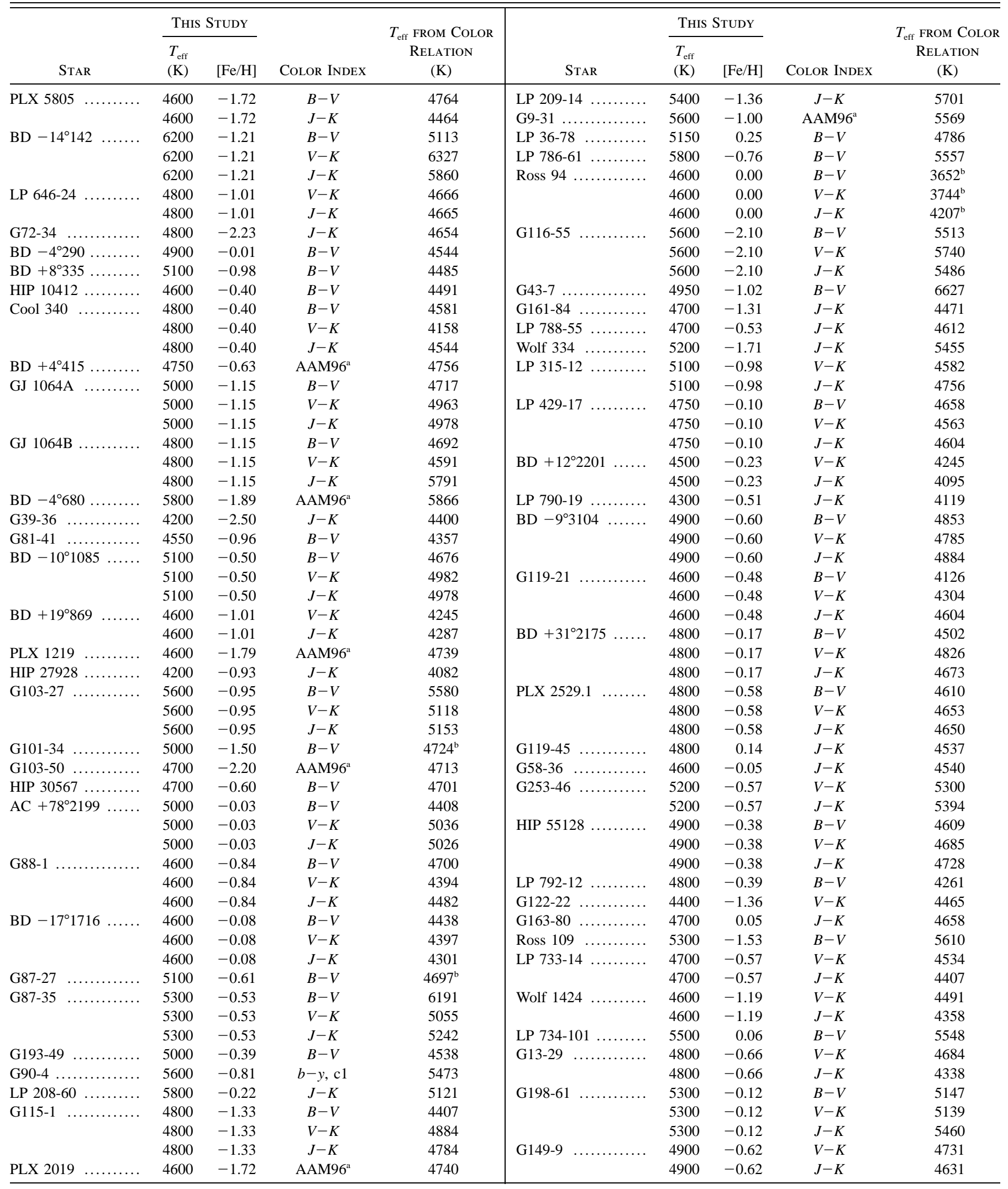


TABLE 3 (Continued)

\begin{tabular}{|c|c|c|c|c|c|c|c|c|c|}
\hline StAR & \multicolumn{2}{|c|}{ THIS STUdY } & COLOR INDEX & $\begin{array}{c}T_{\text {eff }} \text { FROM COLOR } \\
\text { RELATION } \\
(\mathrm{K})\end{array}$ & STAR & \multicolumn{2}{|c|}{ THIS StUdy } & COLOR INDEX & $\begin{array}{c}T_{\text {eff }} \text { FROM COLOR } \\
\text { RELATION } \\
(\mathrm{K})\end{array}$ \\
\hline \multirow[t]{3}{*}{ G149-18 $\ldots \ldots \ldots \ldots$} & 5000 & -0.03 & $B-V$ & 4206 & & 5800 & -1.79 & $V-K$ & 5611 \\
\hline & 5000 & -0.03 & $V-K$ & 4643 & & 5800 & -1.79 & $J-K$ & 5491 \\
\hline & 5000 & -0.03 & $J-K$ & 4650 & LP 747-18 & 5800 & -0.95 & $B-V$ & 5380 \\
\hline & 4730 & -0.71 & $J-K$ & $4565^{\mathrm{b}}$ & LP 447-34 & 4800 & -0.35 & $B-V$ & 5136 \\
\hline & 4730 & -0.71 & ${\text { AAM } 96^{\mathrm{a}}}$ & $4541^{\mathrm{c}}$ & & 4800 & -0.35 & $V-K$ & 4699 \\
\hline \multirow[t]{3}{*}{$\mathrm{BD}+68^{\circ} 714$} & 4900 & -0.05 & $B-V$ & 4424 & & 4800 & -0.35 & $J-K$ & 4760 \\
\hline & 4900 & -0.05 & $V-K$ & 4797 & G19-25 & 4900 & -2.01 & AAM96 ${ }^{\mathrm{a}}$ & 4977 \\
\hline & 4900 & -0.05 & $J-K$ & 4952 & $\mathrm{BD}-8^{\circ} 4501$ & 5800 & -1.80 & AAM96 ${ }^{\mathrm{a}}$ & 5682 \\
\hline Ross 466 & 5000 & -0.86 & $J-K$ & 4784 & G204-47 .... & 5200 & -0.88 & $B-V$ & 4923 \\
\hline & 4900 & -1.30 & $V-K$ & 4686 & G207-23 & 5000 & -1.90 & $B-V$ & 4798 \\
\hline & 4900 & -1.30 & $J-K$ & 4813 & & 5000 & -1.90 & $V-K$ & 5279 \\
\hline \multirow{3}{*}{$\mathrm{BD}+30^{\circ} 2490 \ldots \ldots$} & 4600 & -0.18 & $B-V$ & 4005 & & 5000 & -1.90 & $J-K$ & 5176 \\
\hline & 4600 & -0.18 & $V-K$ & 4185 & HD 181007 & 4700 & -1.93 & $B-V$ & $4659^{b}$ \\
\hline & 4600 & -0.18 & $J-K$ & 4442 & & 4700 & -1.93 & $V-K$ & $4676^{\mathrm{b}}$ \\
\hline G239-12 & 5800 & -2.62 & $B-V$ & 5460 & & 4700 & -1.93 & $J-K$ & $4887^{b}$ \\
\hline HIP $70152 \ldots \ldots \ldots$ & 4800 & -0.05 & $B-V$ & 4446 & LP 752-18 & 5800 & -1.00 & $B-V$ & 6055 \\
\hline \multirow[t]{3}{*}{ LP $500-92 \ldots \ldots \ldots$} & 4500 & -0.38 & $B-V$ & 3765 & G23-14 $\ldots$. & 5000 & -1.51 & $b-y$ & $4958^{b}$ \\
\hline & 4500 & -0.38 & $V-K$ & 4184 & LP 575-39 ... & 4700 & -0.42 & $B-V$ & 4100 \\
\hline & 4500 & -0.38 & $J-K$ & 4294 & LP $575-40$ & 4800 & -0.10 & $B-V$ & 4349 \\
\hline \multirow[t]{2}{*}{ LP $175-8$} & 4900 & -0.31 & $B-V$ & 4490 & LP $815-43$ & 6400 & -2.70 & $B-V$ & 5193 \\
\hline & 4900 & -0.31 & $V-K$ & 4678 & & 6400 & -2.70 & $V-K$ & 6509 \\
\hline \multirow[t]{3}{*}{ LP $330-7 \quad \ldots \ldots \ldots \ldots$} & 4800 & -0.22 & $B-V$ & 4087 & Ross 242 & 4700 & -0.94 & $B-V$ & 4680 \\
\hline & 4800 & -0.22 & $V-K$ & 4743 & & 4700 & -0.94 & $V-K$ & 4682 \\
\hline & 4800 & -0.22 & $J-K$ & 4708 & & 4700 & -0.94 & $J-K$ & 4646 \\
\hline \multirow[t]{3}{*}{ G202-57 } & 4900 & -1.05 & $B-V$ & 4917 & LP 702-79 & 4800 & 0.05 & $V-K$ & 4605 \\
\hline & 4900 & -1.05 & $V-K$ & 4617 & & 4800 & 0.05 & $J-K$ & 4331 \\
\hline & 4900 & -1.05 & $J-K$ & 4612 & HIP 115664 & 4600 & -0.90 & $V-K$ & 4370 \\
\hline G17-25 & 4950 & -1.46 & AAM96 ${ }^{\mathrm{a}}$ & 4966 & & 4600 & -0.90 & $J-K$ & 4411 \\
\hline \multirow[t]{2}{*}{$\mathrm{BD}-14^{\circ} 4454$} & 4600 & -0.95 & $V-K$ & 4474 & $\mathrm{BD}+28^{\circ} 4634 \ldots \ldots$ & 5000 & -0.30 & $B-V$ & 4515 \\
\hline & 4600 & -0.95 & $J-K$ & 4334 & & 5000 & -0.30 & $V-K$ & 4825 \\
\hline LP $445-55 \ldots \ldots \ldots$ & 5200 & -1.80 & $V-K$ & 5807 & & 5000 & -0.30 & $J-K$ & 4712 \\
\hline & 5200 & -1.80 & $J-K$ & 5420 & & & & & \\
\hline
\end{tabular}

Note.-Comparison of $T_{\text {eff }}$ derived in this study with $T_{\text {eff }}$ calculated from Alonso et al. 1996b. Photometry from the following sources: $B$ and $V$ taken from the Guide Star Catalog II, $J$ and $K$ taken from the Two Micron All Sky Survey (2MASS; Skrutskie et al. 1997), and $b-y$ and c1 taken from Schuster et al. 1993.

${ }^{\mathrm{a}} T_{\text {eff }}$ determined from direct application of the IRFM.

${ }^{\mathrm{b}}$ Giant star; $T_{\text {eff }}$ derived from Alonso et al. 1999b relation.

${ }^{\mathrm{c}}$ Giant star assumed to be a dwarf.

REFERENCES.-AAM96 = Alonso et al. 1996a; AAM99 = Alonso et al. 1999a. 


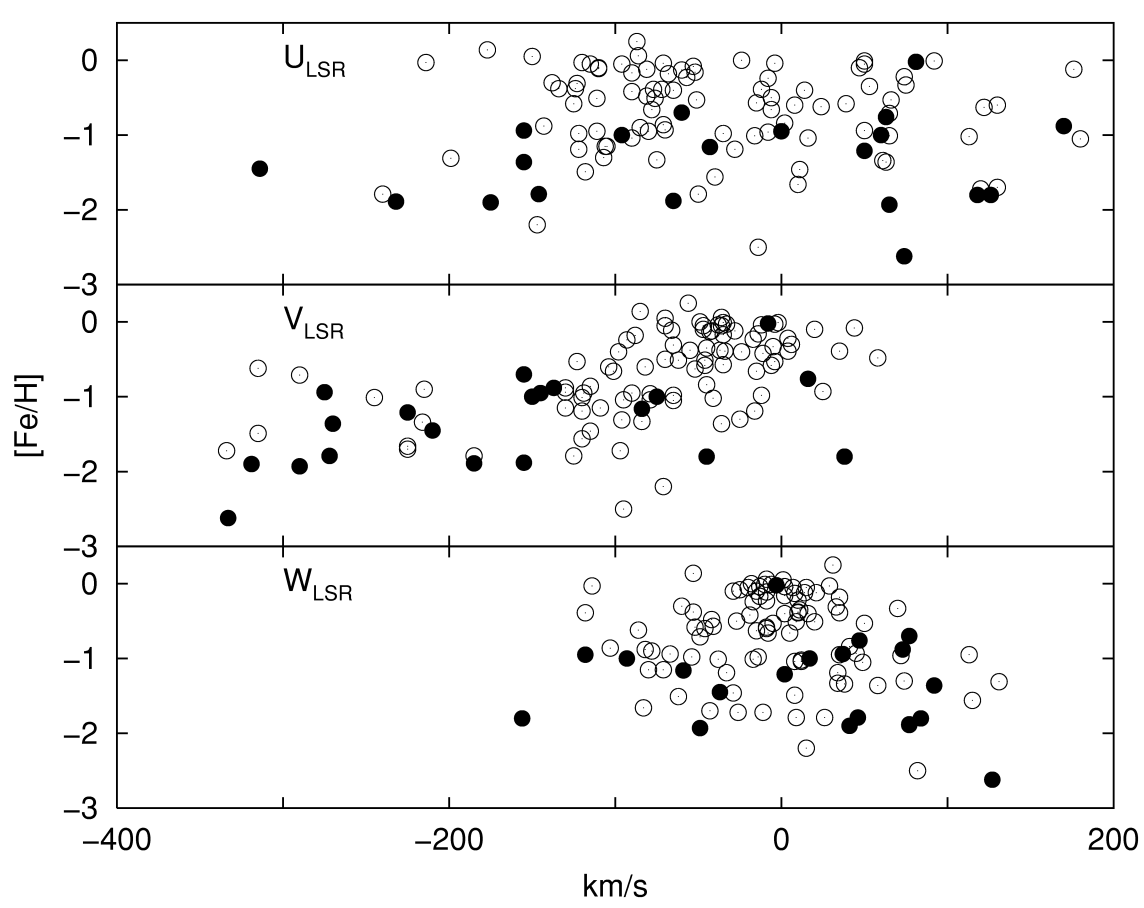

FIG. 7.-Galactic space-velocity $U, V$, and $W$ vs. $[\mathrm{Fe} / \mathrm{H}]$, where $U, V$, and $W$ are relative to the LSR. Filled circles represent stars that have neither MgH nor TiO bands in their spectra. Only stars that have $\left(\sigma_{U}+\sigma_{V}+\sigma_{W}\right) /(|U|+|V|+|W|)<0.7$ are shown, 117 out of 134. A considerable fraction of the sample noticeably lag the LSR $\left(V<-50 \mathrm{~km} \mathrm{~s}^{-1}\right)$.

\subsection{Metallicity}

Figure 8 shows the number of stars versus metallicity for this study, the Carney et al. (1994) sample, and the Ryan \& Norris (1991) sample. It is clear that our sample is considerably smaller than the Ryan and Carney studies. The distributions are similar between the Carney sample and our study, which is rather interesting. Our complete list of NLTT candidates comprised 4445 stars of which we observed some 230 stars. We derived stellar parameters for 134 stars and found 27 with $[\mathrm{Fe} / \mathrm{H}] \leq-1.50$, with several having prior metallicity estimates. Presumably, if we were to observe the remaining $4000+$ candidates, we would find $\sim 500$ stars with $[\mathrm{Fe} / \mathrm{H}] \leq-1.50$. The distribution of the Ryan sample differs from this study and from the Carney study. The Ryan sample peaks at a lower metallicity where this is simply a selection effect. The original Ryan (1989) sample provided broadband photometry where metal-poor candidates were identified by the ultraviolet excess. The stars reobserved in Ryan \& Norris (1991) were those stars identified in Ryan (1989) as having an ultraviolet excess corresponding to $[\mathrm{Fe} / \mathrm{H}]<-1.2$, that is, $\delta(U-B)_{0.6}>0.2$.

For the 27 stars we observed with $[\mathrm{Fe} / \mathrm{H}] \leq-1.50$, there were 11 sufficiently cool to provide $\mathrm{MgH}$ features. These were the cool subdwarfs with molecular features that we were interested in finding. Again, if were to observe the remaining $4000+$ NLTT candidates, there would presumably be $\sim 200$ such objects.
In our sample, the lack of cool subdwarfs with $[\mathrm{Fe} / \mathrm{H}] \leq-2$ could be attributed to two reasons. First, the volume we are sampling may not be large enough, and second, our ability to identify genuine subdwarfs may be inefficient. Regarding the volume we are sampling, according to the Girardi et al. (2000) isochrones, for stars with $T_{\text {eff }}=4500 \mathrm{~K}$ and $[\mathrm{Fe} / \mathrm{H}]=-1.68$, our $m_{R} \simeq V=13$ limit corresponds to a maximum distance of merely $\sim 75 \mathrm{pc}$. This volume further decreases when we consider even more metal poor stars. More importantly, applying our magnitude limit to a star with $T_{\text {eff }}=5500 \mathrm{~K}$ and $[\mathrm{Fe} / \mathrm{H}]=$ -1.68 corresponds to a maximum distance of $\sim 220 \mathrm{pc}$. This represents a volume 27 times larger than for our cooler targets. Carney et al. (1994) considered stars as faint as $V=16$, which, combined with the intrinsic brightness of warmer targets, results in a considerably larger volume. Obviously the number of cool metal-poor stars in a particular volume increases as the volume increases. Regarding the efficiency of identifying candidate subdwarfs, recent work by Salim \& Gould (2002) provides more accurate $V-J$ photometry for NLTT stars based on 2MASS infrared photometry and USNO-A optical photometry. Combining the precise photometry with the proper motions generates a reduced proper-motion diagram with well-defined white dwarf, subdwarf, and disk dwarf populations. From this revised NLTT catalog and proper-motion diagram, we expect that these subdwarf candidates will contain a considerably higher fraction of genuine subdwarfs than our original list of 


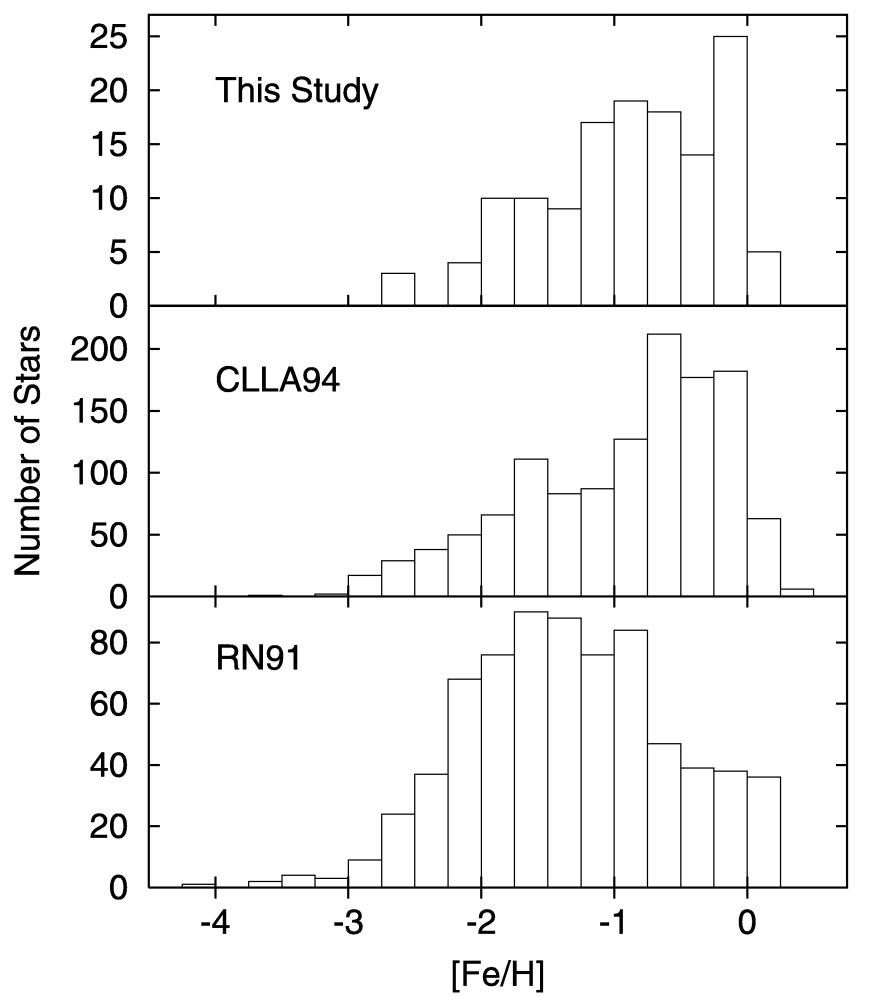

FIG. 8.-Number of stars vs. metallicity for this study (top panel), the Carney et al. (1994) study (middle panel), and the Ryan \& Norris (1991) study (bottom panel). Note the similar distributions between this study and the Carney work. The Ryan distribution peaks at a lower metallicity since their sample consists of stars identified as having an ultraviolet excess corresponding to $[\mathrm{Fe} / \mathrm{H}]<-1.2$.

candidates. Observations of candidate subdwarfs selected from the Salim \& Gould (2002) $V-J$ reduced proper-motion diagram will be made.

Alternatively, we could consider what volume is needed to obtain a large number of stars with $-3<[\mathrm{Fe} / \mathrm{H}]<-2$ and 4000 $\mathrm{K}<T_{\text {eff }}<4500 \mathrm{~K}$. Our observed NLTT candidates provided just one star within the desired range of parameters, G39-36 $\left(T_{\text {eff }}=4200 \mathrm{~K},[\mathrm{Fe} / \mathrm{H}]=-2.5\right)$. From the Yi et al. (2001) isochrones, we estimate that G39-36 lies at a distance of 27 pc from the Sun. Given the stellar parameters of G39-36, our $V=13$ magnitude limit represents a maximum volume of merely 36 pc. Since we observed around 200 of the 4445 NLTT candidates, perhaps there are 10-20 cool subdwarfs among our list of NLTT candidates. If we conservatively estimate that there are five cool subdwarfs $\left(-3<[\mathrm{Fe} / \mathrm{H}]<-2,4000 \mathrm{~K}<T_{\text {eff }}<\right.$ $4500 \mathrm{~K}$ ) within a radius of $50 \mathrm{pc}$ around the Sun, then we expect around $300 \mathrm{cool}$ subdwarfs within a radius of $200 \mathrm{pc}$. A search for subdwarfs similar to G39-36 out to a distance of $200 \mathrm{pc}$ would require a magnitude limit of $V \simeq 17$. Such a limit implies a low-resolution, low-S/N search technique or a photometric search to weed out the intruders.

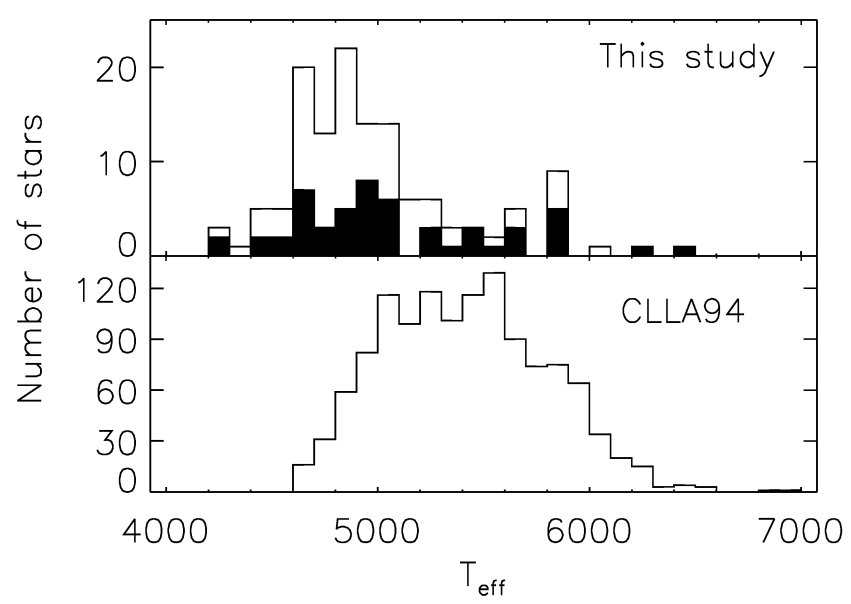

FIG. 9.-Number of stars vs. $T_{\text {eff }}$ for this study (top panel) and the Carney et al. (1994) study (bottom panel). In the top panel, the filled histogram represents the distribution of stars with $[\mathrm{Fe} / \mathrm{H}] \leq-1.0$. The distribution for our sample peaks at lower values of $T_{\text {eff }}$ than the Carney sample. This was expected since we deliberately selected cooler color classes.

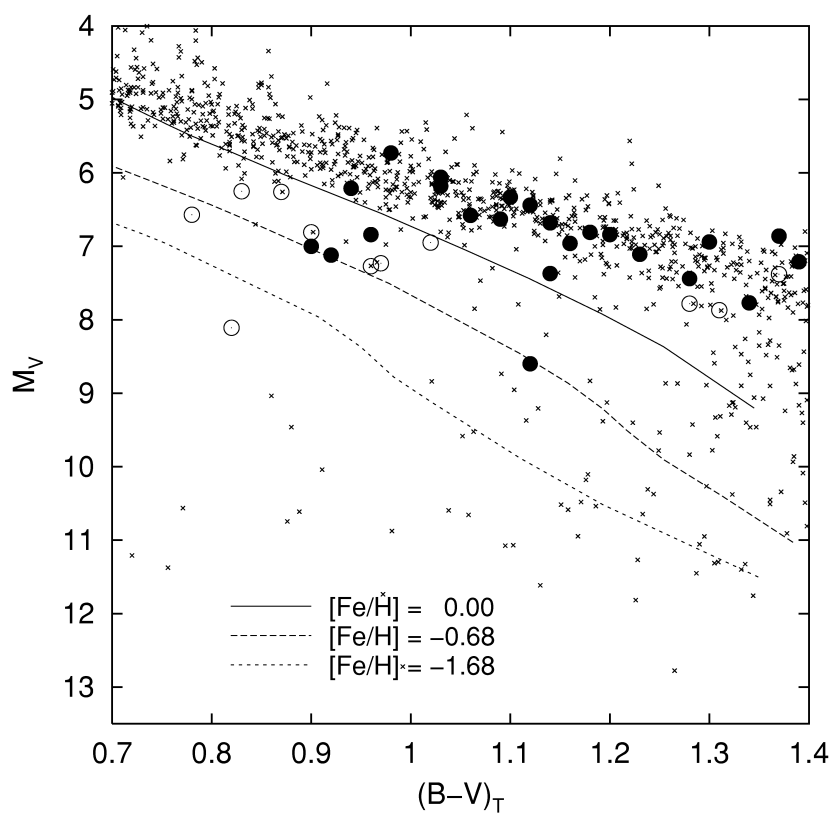

FIG. 10. $-M_{V},(B-V)_{\mathrm{T}}$ color-magnitude diagram with crosses marking Hipparcos stars with $\sigma_{\pi} / \pi<15$ and $\pi>32$ mas. Closed circles represent observed stars with $[\mathrm{Fe} / \mathrm{H}] \geq-1.0$, while open circles represent $[\mathrm{Fe} / \mathrm{H}]<-1.0$. Girardi et al. (2000) isochrones using Johnson $(B-V)$ for $[\mathrm{Fe} / \mathrm{H}]=0.0,-0.68,-1.68$ are overplotted. $(B-V)_{\mathrm{T}}$ is increasingly redder than the Johnson $(B-V)$ as the stars get cooler, as noted by Reid et al. (2001). 


\subsection{Hipparcos}

Having observed a number of cool stars contained in the Hipparcos catalog, we can comment upon the results of Reid et al. (2001). The majority of Hipparcos stars were selected by their large reduced proper motion from the NLTT catalog rather than the position in the color-magnitude diagram. Reid et al. (2001) claimed that in a color-magnitude diagram produced from Hipparcos parallaxes, the majority of stars with $(B-V) \leq 0.8$ situated below the solar-metallicity main sequence were incorrectly positioned as a result of errors in the colors. These stars, located where subdwarfs ought to lie, were metal-rich disk dwarfs rather than subdwarfs. Reid et al. (2001) also showed that the Tycho colors $(B-V)_{\mathrm{T}}$ are redder than the Johnson $(B-V)$ colors. In Figure 10, we take the Tycho colors and Hipparcos parallaxes to produce a color-magnitude diagram overlaying the stars we observed. Nearby stars $(\pi>32$ mas) with accurate parallaxes $\left(\sigma_{\pi} / \pi<0.15\right)$ are included to represent the disk population, $[\mathrm{Fe} / \mathrm{H}] \simeq 0$. Our few observations confirm and extend the Reid et al. (2001) findings in the regime $0.8<B-V<1.4$. Metal-poor stars with $[\mathrm{Fe} / \mathrm{H}]<-1.0$ are located among disk stars. Also, metal-rich stars with $[\mathrm{Fe} / \mathrm{H}]>-1.0$ are offset from the disk main sequence. The effect of the redder Tycho colors is evident as the disk main sequence is systematically offset from the Girardi et al. (2000) $[\mathrm{Fe} / \mathrm{H}]=0$ isochrone. We intend to observe additional Hipparcos subdwarf candidates in the range $0.8<(B-V)_{\mathrm{T}}<1.2$ not only to find cool subdwarfs but to further test the findings made by Reid et al. (2001).

\section{CONCLUDING REMARKS}

We present stellar parameters for 134 candidate subdwarfs selected by their reduced proper motion, offset from the solarmetallicity main sequence, or from the literature. Our goal was to provide a large database of cool subdwarfs. Our selection criteria were successful in identifying cool stars (69 with $T_{\text {eff }}<$ $4800 \mathrm{~K})$ and metal-poor stars $(27$ with $[\mathrm{Fe} / \mathrm{H}] \leq-1.50)$. Of our sample, 11 stars were sufficiently cool to provide measurable $\mathrm{MgH}$ lines with $[\mathrm{Fe} / \mathrm{H}]<-1.50$. Armed with a sample of cool subdwarfs, we can begin to exploit their unique qualities. For a subset of our sample, we have measured the $\mathrm{Mg}$ isotopic abundance ratios and compared the observed trends with predictions from models of Galactic chemical evolution. This work will be presented in a future paper. We also intend to make further observations of candidate subdwarfs. Targets will be selected from the revised NLTT catalog (Salim \& Gould 2002). Cool Hipparcos subdwarf candidates will be observed, and we anticipate that Christlieb and collaborators will identify cool subdwarfs in the Hamburg/ESO survey data.

We thank George Preston for helpful comments and the staff of the Hobby-Eberly telescope for service observations of our targets. We acknowledge support via the grant F-634 from the Robert A. Welch Foundation of Houston, Texas. This research has made use of the SIMBAD database, operated at CDS, Strasbourg, France, and NASA's Astrophysics Data System.

\section{REFERENCES}

Alibés, A., Labay, J., \& Canal, R. 2001, A\&A, 370, 1103

Alonso, A., Arribas, S., \& Martínez-Roger, C. 1996a, A\&AS, 117, 227

$$
\begin{aligned}
& \text { 1996b, A\&A, 313, } 873 \\
& \text { 1999a, A\&AS, 139, } 335 \\
& \text { 1999b, A\&AS, 140, } 261
\end{aligned}
$$

Beers, T. C., Preston, G. W., \& Shectman, S. A. 1985, AJ, 90, 2089 1992, AJ, 103, 1987

Beers, T. C., Rossi, S., Norris, J. E., Ryan, S. G., \& Shefler, T. 1999, AJ, 117, 981

Bond, H. E. 1970, ApJS, 22, 117 1980, ApJS, 44, 517

Carney, B. W., \& Latham, D. W. 1987, AJ, 93, 116

Carney, B. W., Latham, D. W., Laird, J. B., \& Aguilar, L. A. 1994, AJ, 107, 2240

Carney, B. W., Wright, J. S., Sneden, C., Laird, J. B., Aguilar, L. A., \& Latham, D. W. 1997, AJ, 114, 363

Christlieb, N. 2000, Ph.D. thesis, Univ. Hamburg

Clementini, G., Gratton, R. G., Carretta, E., \& Sneden, C. 1999, MNRAS, 302, 22

Dehnen, W., \& Binney, J. J. 1998, MNRAS, 298, 387

Edvardsson, B., Andersen, J., Gustafsson, B., Lambert, D. L., Nissen, P. E., \& Tomkin, J. 1993, A\&A, 275, 101

Eggen, O. J. 1987, AJ, 93, 393 . 1998, AJ, 115, 2397
ESA. 1997, The Hipparcos and Tycho Catalogues, VizieR Online Data Catalog

Fuchs, B., \& Jahreiß, H. 1998, A\&A, 329, 81

Fulbright, J. P. 2000, AJ, 120, 1841

Gay, P. L., \& Lambert, D. L. 2000, ApJ, 533, 260

Girardi, L., Bressan, A., Bertelli, G., \& Chiosi, C. 2000, A\&AS, 141, 371

Gizis, J. E. 1997, AJ, 113, 806

Goswami, A., \& Prantzos, N. 2000, A\&A, 359, 191

Grevesse, N., \& Sauval, A. J. 1999, A\&A, 347, 348

Harlow, J. J. B., Ramsey, L. W., Andersen, D. R., Fleig, J. D., Rhoads, B. T., \& Engel, L. G. 1996, BAAS, 28, 1324

Hauschildt, P. H., Allard, F., \& Baron, E. 1999, ApJ, 512, 377

Johnson, D. R. H., \& Soderblom, D. R. 1987, AJ, 93, 864

Kurucz, R. 1993, Kurucz CD-ROM 13, ATLAS9 Stellar Atmosphere Programs and $2 \mathrm{~km} / \mathrm{s}$ Grid (Cambridge: Smithsonian Astrophys. Obs.), 13

Lambert, D. L., Heath, J. E., Lemke, M., \& Drake, J. 1996, ApJS, 103,183

Luyten, W. J. 1979, New Luyten Catalogue of Stars with Proper Motions Larger than Two Tenths of an Arcsecond, Vols. 1-4 (Minneapolis: Univ. Minnesota)

- 1980, New Luyten Catalogue of Stars with Proper Motions Larger than Two Tenths of an Arcsecond, Vols. 1-4 (Minneapolis: Univ. Minnesota) 


\section{YONG \& LAMBERT}

Luyten, W. J., \& Hughes, H. S. 1980, Proper Motion Survey with the Forty-Eight Inch Schmidt Telescope. LV. First Supplement to the NLTT Catalogue (Minneapolis: Univ. Minnesota)

McWilliam, A., Preston, G. W., Sneden, C., \& Searle, L. 1995, AJ, 109,2757

Morrison, H. L., Olszewski, E. W., Mateo, M., Norris, J. E., Harding, P., Dohm-Palmer, R. C., \& Freeman, K. C. 2001, AJ, 121, 283

Norris, J. 1986, ApJS, 61, 667

Primas, F., Molaro, P., Bonifacio, P., \& Hill, V. 2000, A\&A, 362, 666

Reid, I. N., van Wyk, F., Marang, F., Roberts, G., Kilkenny, D., \& Mahoney, S. 2001, MNRAS, 325, 931

Ryan, S. G. 1989, AJ, 98, 1693

Ryan, S. G., \& Norris, J. E. 1991, AJ, 101, 1835

Salim, S., \& Gould, A. 2002, ApJ, 575, L83
Schuster, W. J., Parrao, L., \& Contreras Martinez, M. E. 1993, A\&AS, 97, 951

Skrutskie, M. F., et al. 1997, in Astrophysics and Space Science Library 210, The Impact of Large Scale Near-IR Sky Surveys, ed. F. Garzón et al. (Dordrecht: Kluwer), 25

Sneden, C. A. 1973, Ph.D. thesis, Univ. Texas

Thévenin, F., \& Idiart, T. P. 1999, ApJ, 521, 753

Timmes, F. X., Woosley, S. E., \& Weaver, T. A. 1995, ApJS, 98, 617

Tomkin, J., \& Lambert, D. L. 1999, ApJ, 523, 234

Tull, R. G., MacQueen, P. J., Sneden, C., \& Lambert, D. L. 1995, PASP, 107, 251

van Altena, W. F., Lee, J. T., \& Hoffleit, D. 1995, Yale Trigonometric Parallaxes, VizieR Online Data Catalog

Yi, S., Demarque, P., Kim, Y., Lee, Y., Ree, C. H., Lejeune, T., \& Barnes, S. 2001, ApJS, 136, 417 